\title{
THE RISE AND FALL OF TYPE Ia SUPERNOVA LIGHT CURVES IN THE SDSS-II SUPERNOVA SURVEY
}

\author{
Brian T. Hayden ${ }^{1}$, Peter M. Garnavich ${ }^{1}$, Richard Kessler ${ }^{2,3}$, Joshua A. Frieman ${ }^{2,4,5}$, Saurabh W. Jha ${ }^{6,7}$, \\ Bruce Bassett $^{8,9}$, David Cinabro $^{10}$, Benjamin Dilday ${ }^{7}$, Daniel Kasen ${ }^{11,20}$, John Marriner ${ }^{12}$, Robert C. Nichol ${ }^{13}$, \\ Adam G. Riess ${ }^{14,15}$, Masao Sako ${ }^{16}$, Donald P. Schneider ${ }^{17}$, Mathew Smith ${ }^{8}$, and Jesper Sollerman ${ }^{18,19}$ \\ ${ }^{1}$ University of Notre Dame, 225 Nieuwland Science Hall, Notre Dame, IN 46556, USA \\ ${ }^{2}$ Kavli Institute for Cosmological Physics, University of Chicago, Chicago, IL 60637, USA \\ ${ }^{3}$ Enrico Fermi Institute, University of Chicago, Chicago, IL 60637, USA \\ ${ }^{4}$ Department of Astronomy and Physics, University of Chicago, Chicago, IL 60637, USA \\ ${ }^{5}$ Center for Particle Astrophysics, Fermi National Accelerator Laboratory, Batavia, IL 60510, USA \\ ${ }^{6}$ Kavli Institute for Particle Astrophysics and Cosmology, Stanford University, Stanford, CA 94305-4060, USA \\ ${ }_{7}^{7}$ Department of Physics and Astronomy, Rutgers University, 136 Frelinghuysen Road, Piscataway, NJ 08854, USA \\ ${ }^{8}$ Department of Mathematics and Applied Mathematics, University of Cape Town, Rondebosch 7701, South Africa \\ ${ }^{9}$ South African Astronomical Observatory, Cape Town, South Africa \\ ${ }^{10}$ Department of Physics and Astronomy, Wayne State University, Detroit, MI, USA \\ ${ }^{11}$ Department of Astronomy and Astrophysics, University of California, Santa Cruz, CA 95064, USA \\ 12 Center for Particle Astrophysics, Fermi National Accelerator Laboratory, P.O. Box 500, Batavia, IL 60510, USA \\ 13 Institute of Cosmology \& Gravitation, University of Portsmouth, Portsmouth PO1 3FX, UK \\ ${ }^{14}$ Space Telescope Science Institute, 3700 San Martin Drive, Baltimore, MD 21218, USA \\ 15 Department of Physics and Astronomy, Johns Hopkins University, 3400 North Charles Street, Baltimore, MD 21218, USA \\ ${ }^{16}$ Department of Physics and Astronomy, University of Pennsylvania, 209 South 33rd Street, Philadelphia, PA 19104, USA \\ ${ }_{17}$ Department of Astronomy and Astrophysics, Pennsylvania State University, 525 Davey Laboratory, University Park, PA 16802, USA \\ ${ }^{18}$ Dark Cosmology Centre, Niels Bohr Institute, University of Copenhagen, Juliane Maries Vej 30, DK-2100 Copenhagen O, Denmark \\ 19 Department of Astronomy, The Oskar Klein Centre, Stockholm University, 10691 Stockholm, Sweden \\ Received 2009 September 30; accepted 2010 January 19; published 2010 March 2
}

\begin{abstract}
We analyze the rise and fall times of Type Ia supernova (SN Ia) light curves discovered by the Sloan Digital Sky Survey-II (SDSS-II) Supernova Survey. From a set of 391 light curves $k$-corrected to the rest-frame $B$ and $V$ bands, we find a smaller dispersion in the rising portion of the light curve compared to the decline. This is in qualitative agreement with computer models which predict that variations in radioactive nickel yield have less impact on the rise than on the spread of the decline rates. The differences we find in the rise and fall properties suggest that a single "stretch" correction to the light curve phase does not properly model the range of SN Ia light curve shapes. We select a subset of 105 light curves well observed in both rise and fall portions of the light curves and develop a "2-stretch" fit algorithm which estimates the rise and fall times independently. We find the average time from explosion to $B$-band peak brightness is $17.38 \pm 0.17$ days, but with a spread of rise times which range from 13 days to 23 days. Our average rise time is shorter than the 19.5 days found in previous studies; this reflects both the different light curve template used and the application of the 2-stretch algorithm. The SDSS-II supernova set and the local SNe Ia with well-observed early light curves show no significant differences in their average rise-time properties. We find that slow-declining events tend to have fast rise times, but that the distribution of rise minus fall time is broad and single peaked. This distribution is in contrast to the bimodality in this parameter that was first suggested by Strovink from an analysis of a small set of local SNe Ia. We divide the SDSS-II sample in half based on the rise minus fall value, $t_{r}-t_{f} \leqslant 2$ days and $t_{r}-t_{f}>2$ days, to search for differences in their host galaxy properties and Hubble residuals; we find no difference in host galaxy properties or Hubble residuals in our sample.
\end{abstract}

Key words: methods: data analysis - supernovae: general - white dwarfs

Online-only material: color figure

\section{INTRODUCTION}

Type Ia supernovae (SNe Ia) are bright stellar explosions important for their role as distance indicators. SN Ia distances have been used to constrain the value of the Hubble constant (Jha et al. 1998; Freedman et al. 2001), and they also showed that our universe has a lower than critical matter density (Garnavich et al. 1998a; Perlmutter et al. 1998). Riess et al. (1998) and Perlmutter et al. (1999) used distant SNe Ia to show that the universe currently has an accelerating rate of expansion implying a "dark" energy component. As more SN Ia observations have confirmed this result (Knop et al. 2003; Tonry et al. 2003; Barris et al. 2006; Riess et al. 2004), the focus has shifted to constraining

\footnotetext{
${ }^{20}$ Hubble fellow.
}

the properties of dark energy (Garnavich et al. 1998b; Astier et al. 2006; Riess et al. 2007; Wood-Vasey et al. 2007; Miknaitis et al. 2007; Eisenstein et al. 2007). Systematic uncertainties in measuring dark energy parameters with supernovae now dominate over statistical errors (Kessler et al. 2009a) and a better understanding of supernova physics may help to constrain dark energy properties using SNe Ia.

It is widely accepted that $\mathrm{SNe}$ Ia are the result of thermonuclear explosions of carbon-oxygen white dwarfs (WDs), but the nature of the progenitor remains uncertain. In most models, the explosion occurs when the WD nears the Chandrasekhar limit by gaining mass from a binary companion. This mass gain is achieved either by single-degenerate (SD) mass transfer or WD coalescence in a double-degenerate (DD) scenario. For a comprehensive review of these models, see Livio (2000, 
p. 33). Observers have attempted to distinguish between the two models, with conflicting results. Howell et al. (2006) found that the extremely luminous supernova SNLS-03D3bb (SN 2003fg) had low ejecta velocity that could have resulted from a superChandrasekhar progenitor. The high total mass and large nickel yield suggested that SNLS-03D3bb was the product of a DD merger. Hicken et al. (2007) found that SN 2006gz had attributes consistent with the DD model. Its spectrum showed significant amounts of unburned carbon, and a low silicon velocity at early phases. The very broad light curve implies a large yield of radioactive nickel as expected in the DD model (Hicken et al. 2007), although late-time observations show only weak iron emission lines (Maeda et al. 2009). Even though the SD model is generally accepted as the most plausible (Livio 2000, p. 33), there is evidence that it might not be the complete story (Hicken et al. 2007; Pritchet et al. 2008). Some mixture of progenitors may be producing events with subtle differences in character. The explosion rates for these progenitor systems will depend on the age and the star formation history of the parent population, and the ratio of the different progenitors may not be constant through the history of the universe (Hamuy et al. 1996b; Gallagher et al. 2005, 2008; Sullivan et al. 2006; Mannucci et al. 2006).

Understanding the origin of the Phillips relation (the correlation between light curve shape and peak luminosity; Phillips 1993; Kasen \& Woosley 2007) and its dispersion is critical to improving SNe Ia as reliable distance indicators. Timmes et al. (2004, p. 179) argued that radioactive nickel yield should be determined by progenitor metallicity, but the age of the host galaxy stellar population appears to control the mass of ${ }^{56} \mathrm{Ni}$ (Hamuy et al. 1996b; Gallagher et al. 2005; Sullivan et al. 2006). Progenitor metallicity may play a secondary role (Gallagher et al. 2008) or have no significant effect on radioactive yield (Howell et al. 2009). Recent models by Woosley et al. (2007) show that variations in kinetic energy (KE), metallicity, and mixing between burning layers provide light curves beyond the range of the observed Phillips relation, implying that not all combinations of variables are found in real SNe Ia.

SN Ia luminosity and light curve shape may be influenced by the physics of the explosions. There is a consensus that normal SNe Ia result from a detonation (supersonic burning) of much of the progenitor WD, but it likely begins as a deflagration (subsonic fusion front) to allow expansion and some burning at low densities. This model was first introduced by Khokhlov (1991); Hoeflich \& Khokhlov (1996) showed that the model matched the observed light curves and spectra very well. In the "delayed detonation" scenario, the timing of the transition from deflagration to detonation provides a natural means of varying the radioactive nickel yield and generating the observed range of luminosities and decline rates (Arnett \& Livne 1994). Deflagrations tend to be very asymmetric, and if the asymmetries survive the detonation then viewing angle can result in perceived variations even for similar explosions (Kasen \& Plewa 2007).

Study of the early-time light curve may be important in diagnosing the progenitor problem and explosion physics (Hoeflich $\&$ Khokhlov 1996). The work by Riess et al. (1999) to constrain the early light curves of local supernovae established the decline-rate-corrected average rise time of $19.5 \pm 0.2$ days. Here, "rise time" is defined as the time elapsed from explosion to peak $B$-band flux. Aldering et al. (2000) and Goldhaber et al. (2001) demonstrated the consistency between high- and lowredshift rise times, and similar results were found by Conley et al. (2006) with a large set of SNLS (Pritchet et al. 2005) supernovae. Garg et al. (2007) studied the rise times of SNe Ia discovered behind the Large Magellanic Cloud during the SuperMACHO survey lens project and measured a $17.6 \pm 1.3$ day rise time in the equivalent of the $V$ bandpass.

Recently, Strovink (2007) analyzed eight low-redshift SNe Ia with well-observed early light curves from a new perspective. A single "stretch" parameter has commonly been used to describe the full range of $B$ and $V$ light curves by compressing or expanding the time axis around the epoch of peak brightness (Perlmutter et al. 1997). Strovink (2007) decoupled the rise and decline portions of the eight nearby light curves and found that $\mathrm{SNe}$ Ia can have a range of rise times for a given decline rate. His rise-time minus fall-time distribution $\left(t_{r}-t_{f}\right.$; hereafter defined as $\mathrm{RMF}$ ) roughly divided his small supernova sample into two groups, possibly suggesting two progenitors or explosion mechanisms. A larger set of $\mathrm{SNe}$ is required to rigorously test this possibility.

Here, we analyze light curves from the Sloan Digital Sky Survey-II (SDSS-II) Supernova Survey (Frieman et al. 2008) which spectroscopically identified approximately $500 \mathrm{SNe}$ Ia over its three-year lifetime. The SDSS-II Supernova Survey scanned $300 \mathrm{deg}^{2}$ of sky with a cadence as rapid as 2 days between visits (weather and lunar phase often increased the time between observations of the same field), making the survey well suited to an early rise-time study. We also introduce a new fitting method that independently estimates the rise and the fall times of SNe Ia light curves.

\section{DATA}

\subsection{SDSS-II Light Curves}

The SDSS-II Supernova Survey (Frieman et al. 2008) was designed to find and characterize several hundred $\mathrm{SNe}$ Ia at intermediate redshifts in order to fill in the supernova "desert" between the nearby discoveries and the "high- $z$ " events. Mapping the expansion history at $z \approx 0.2$ provides unique tests of cosmological models and constrains systematic errors (Kessler et al. 2009a; Sollerman et al. 2009; Lampeitl et al. 2010). The SDSS-II supernovae also provide a large, uniform, high-quality sample of SNe Ia to study the properties of these explosions. The SDSS-II Supernova Survey operated three campaigns between 2005, 2006, and 2007 September and December using the $2.5 \mathrm{~m}$ telescope (Gunn et al. 2006) at Apache Point Observatory to scan $300 \mathrm{deg}^{2}$ of sky as often as every second night. Template images were subtracted from each new night of data and software scanned for new variable objects. Every candidate supernova was inspected visually to avoid image artifacts and asteroids. Transients with a high probability of being SNe Ia (Sako et al. 2008) were queued for spectrographic observation (Zheng et al. 2008). The SDSS-II SN survey takes advantage of the extensive database of reference images, object catalogs, and photometric calibration compiled by the SDSS (see York et al. 2000 for an overview of the SDSS). The early data release of the SDSS can be found in Stoughton et al. (2002). Data release seven, the final data release of SDSS-II, can be found in Abazajian et al. (2009).

The supernova sample minimizes photometric errors by using a single filter set and a photometric system (Smith et al. 2002) calibrated by the original SDSS project (Fukugita et al. 1996; Gunn et al. 1998). The photometric quality is assessed in Ivezić et al. (2004), and the astrometric calibrations are described in Pier et al. (2003). Specifically, the area of the SDSS-II SN survey has been calibrated to $1 \%$ photometric error (Smith et al. 2002). 

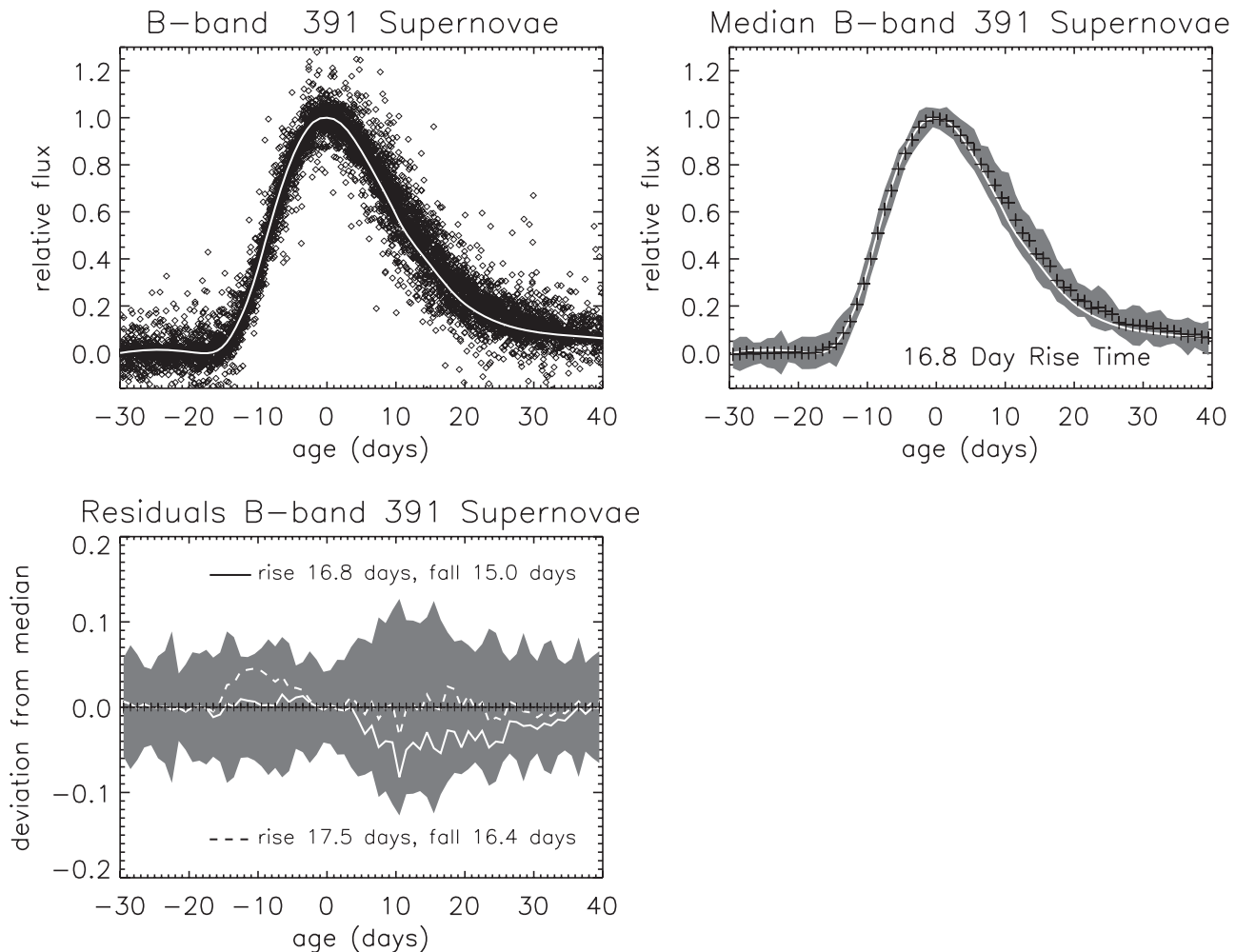

Figure 1. Top left: individual SDSS-II supernova observations plotted in the rest frame and normalized to a peak flux of unity. The light curves were $k$-corrected to $B$ band using SNANA. Overplotted are the MLCS2k2 fiducial light curves with a 16.8 day rise time. Top right: median of all the data points binned by day are plotted $(+)$ and the gray regions show the rms deviations about the median values. The white lines show the MLCS2k2 fiducial curves. Bottom: fluctuations about the median are plotted along with template light curve residuals. This clearly shows the scatter in rise time is significantly less than in the decline portion of the light curve. The solid line shows the MLCS2k2 template with a 16.8 day rise time is an excellent match to the median of the $391 \mathrm{SNe}$ Ia. The dashed line indicates that increasing the rise time by 0.7 days is clearly a poor fit to the median rise data while a decline of 16.4 days is a good match.

The photometric calibration employed several minor telescopes as well; this Monitor Telescope Pipeline (MTPIPE) is described in Tucker et al. (2006).

A software "robot" was developed that automatically reduced CCD observations of standard stars in order to assess photometricity and build data on site conditions at the Apache Point Observatory (Hogg et al. 2001). Using this tool to select the best observing nights, weather and lunar phase extend the time between observations to an average of about 4.5 days (Frieman et al. 2008). The rapid cadence of the SDSS-II SN survey assures that most of the SDSS SNe have well-defined, densely sampled light curves even on the rise portion. The data include pre-explosion flux measurements, so that many of the SDSS-II Supernova Survey Type Ia light curves are among the most wellsampled and well-calibrated light curves that have been studied to date.

The SDSS-II SN survey identified 498 spectroscopically confirmed $\mathrm{SNe}$ Ia (number accurate to the time of our analysis). Five of these are extremely peculiar and are not included in this study. SN 2007qd (C. McClelland 2010, in preparation) and SN 2005hk (Phillips et al. 2007) are categorized as 2002cx-like objects. SN 2002cx showed evidence for a very low expansion velocity compared to "normal" $\mathrm{SNe}$ Ia as well as an unusually low peak luminosity, leading to the hypothesis that it was a pure deflagration event (Branch et al. 2004; Jha et al. 2006). SN 2005gj, SN 7017, and SN 15557 (Prieto et al. 2007) are 2002ic like. SN 2002ic showed a Type Ia spectrum along with strong hydrogen emission lines (Hamuy et al. 2003) suggesting a shock interacting with circumstellar material.

We limit the events to redshift less than 0.4 and require that there be at least one photometric epoch more than 2 days before maximum and more than 5 days after maximum. These requirements result in a sample of 391 supernovae. The photometry is measured using "scene modeling" developed by Holtzman et al. (2008). The Sloan $g, r$, and $i$ photometry has been $k$-corrected to Bessell $B$ and $V$ bands using SNANA (Kessler et al. 2009b; Hsiao et al. 2007). We use the time of maximum obtained by the fitting procedure in SNANA as the initial value for our $\chi^{2}$ minimizing function. The redshift of the $391 \mathrm{SNe}$ Ia ranges from 0.037 to 0.40 with a median redshift of 0.21 .

Figure 1 displays the entire set of $391 B$ and $V$ light curves after estimating the time of maximum, normalizing the peak to unity, and correcting for time dilation. The data were binned in 1 day intervals and the median value calculated for each day, also shown in Figure 1. The MLCS2k2 fiducials are an excellent match to the median light curves, with an extrapolation of 16.8 days. The fading portion of the SDSS-II $B$-band data is brighter than the fiducial, indicating that the supernovae discovered by SDSS-II are, on average, slower than typical lowredshift events. For the $V$ band, the fiducial and the SDSS-II light curves match well. It is clear that the scatter about the median light curve is smaller during the rise than during the decline. The maximum root-mean-square (rms) dispersion for $B$ band in 1 day bins is 0.09 (scaled flux units) on the rise and 0.13 on the decline. The average rms about the median on the rise is 0.069 while on the fall it is 0.091 . This implies a smaller range of rise times than fall times and therefore that the full range of SN Ia light curve shapes cannot be well fitted by a single-stretch parameter.

Kasen (2010) has provided a theoretical prediction of the impact on the light curve of SN Ia due to the interaction of the WD explosion with a potential companion star. It is estimated 
Table 1

Comparison of 2-stretch Method versus Strovink (2007)

\begin{tabular}{|c|c|c|c|c|c|c|}
\hline $\mathrm{SN}$ & $\begin{array}{c}\Delta m_{15}(\mathrm{mag}) \\
\text { Strovink07 }\end{array}$ & $\begin{array}{c}\Delta m_{15}(\mathrm{mag}) \\
\text { 2-stretch }\end{array}$ & $\begin{array}{l}t_{\text {rise }}(\text { days) } \\
\text { Strovink07 }\end{array}$ & $\begin{array}{c}t_{\text {rise }} \text { (days) } \\
2 \text {-stretch }\end{array}$ & $\begin{array}{c}t_{r}-t_{f} \text { (days) } \\
\text { Strovink07 }\end{array}$ & $\begin{array}{c}t_{r}-t_{f} \text { (days) } \\
\text { 2-stretch } \\
\end{array}$ \\
\hline SN 1990N & $0.990 \pm 0.034$ & $1.029 \pm 0.017$ & $20.01 \pm 0.46$ & $19.81 \pm 0.25$ & $4.04 \pm 0.74$ & $4.31 \pm 0.26$ \\
\hline SN 1994D & $1.344 \pm 0.021$ & $1.422 \pm 0.023$ & $15.39 \pm 0.47$ & $15.43 \pm 0.23$ & $2.04 \pm 0.53$ & $2.73 \pm 0.23$ \\
\hline SN 1998aq & $1.042 \pm 0.021$ & $1.089 \pm 0.013$ & $17.52 \pm 0.58$ & $17.13 \pm 0.22$ & $2.03 \pm 0.64$ & $2.20 \pm 0.22$ \\
\hline SN 2001el & $1.168 \pm 0.021$ & $1.080 \pm 0.010$ & $18.00 \pm 0.56$ & $18.22 \pm 0.18$ & $3.57 \pm 0.63$ & $3.21 \pm 0.18$ \\
\hline SN 2002bo & $1.162 \pm 0.031$ & $1.168 \pm 0.031$ & $16.05 \pm 0.37$ & $17.50 \pm 0.19$ & $1.57 \pm 0.53$ & $3.21 \pm 0.21$ \\
\hline SN 2003du & $0.982 \pm 0.021$ & $1.013 \pm 0.010$ & $17.71 \pm 0.35$ & $17.77 \pm 0.17$ & $1.64 \pm 0.44$ & $2.11 \pm 0.17$ \\
\hline SN 2004eo & $1.403 \pm 0.037$ & $1.326 \pm 0.010$ & $16.64 \pm 0.44$ & $16.62 \pm 0.13$ & $3.86 \pm 0.53$ & $3.39 \pm 0.13$ \\
\hline SN 2005cf & $1.068 \pm 0.021$ & $1.045 \pm 0.008$ & $16.62 \pm 0.25$ & $17.31 \pm 0.10$ & $1.35 \pm 0.36$ & $1.97 \pm 0.10$ \\
\hline
\end{tabular}

that approximately $10 \%$ of SN Ia explosions that occur in the SD channel would have light curves that are affected by the shock interaction of the explosion with a binary companion. This effect is not immediately obvious in the SDSS-II from any analysis in this paper; however, an in-depth statistical study is in progress to rigorously test the SDSS-II SN Ia sample for this effect (Hayden 2010).

\subsection{The Low-redshift SN Ia Set}

In order to compare our fitter with the results in Strovink (2007), we applied our 2-stretch fitting method to the same set of eight low- $z$ SNe Ia studied by Strovink. These supernovae are SN 1990N (Lira et al. 1998), SN 1994D (Patat et al. 1996), SN 1998aq (Riess et al. 2005), SN 2001el (Krisciunas et al. 2003), SN 2002bo (Benetti et al. 2004), SN 2003du (Stanishev et al. 2007), SN 2004eo (Pastorello et al. 2007b), and SN 2005cf (Pastorello et al. 2007a). Strovink (2007) combines light curve observations from different studies in his analysis of low- $z$ supernovae, but this has the danger of conflicting calibrations and differences in photometric error estimates. Instead, we limit observations to a single published data set selected based on cadence and number of data points before maximum. Table 1 compares the Strovink and the 2-stretch fitter results. We find very similar values for these supernovae. The average difference between the rise time measured by Strovink (2007) and our 2 -stretch fitter is $-0.23 \pm 0.58$ days (standard deviation) and the difference between fall times is $0.15 \pm 0.47$ days (standard deviation).

\section{2-STRETCH FITTING METHOD}

With a handful of well-observed events, Strovink (2007) found that the rise time of SNe Ia can vary for a fixed decay time. He developed a fitting method called Adaptive QUArtic Algorithm (AQUAA) which employs quartic splines with fuzzy knots to create smooth UBVRI light curves for individual supernovae. The Strovink (2007) fitter is computer intensive and does not work as well with noisy data; both are drawbacks when dealing with the large quantity and diverse quality of SDSS-II supernova data (see Section 2.1).

For the SDSS-II data, we desired a fitting method which maintains the simplicity of fitting light curves with the singlestretch technique, while decoupling the rise and fall portions during the fitting process. Our "2-stretch" method attempts to accomplish this goal by stretching the pre-maximum portion separately from the post-maximum portion of the light curve. Our template curve is required to stay continuous at the joining point: peak brightness. Maximum light has a zero first derivative, making this a natural cutting point for using a 2 -stretch method. A $\chi^{2}$ minimization is performed to determine the four parameters of the fit: rise stretch $\left(s_{r}\right)$, fall stretch $\left(s_{f}\right)$, time of maximum, and peak flux. It is not possible to completely remove the covariance between rise and fall times, because photometric errors and sampling gaps produce uncertainty in the time of maximum which mixes rise and fall times. Fitting simulated light curves with known rise and fall times allows us to estimate the correlation between rise and fall errors.

Our implementation of the fitting method uses $B$ and $V$ fiducial curves generated by MLCS2K2 (Jha et al. 2007) for a typical, $\Delta=0$ supernova. The MLCS method has the advantage of creating a template from a large number of nearby supernovae. However, few of the supernovae used to train MLCS2k2 had light curve information earlier than 10 days before maximum light. For the region earlier than -10 days, we assume a simple expanding fireball model which implies that the flux from the moment of explosion increases as the square of the time (Goldhaber et al. 2001; Riess et al. 1999). We then create a set of possible early-time light curves that all join with the MLCS2k2 fiducial at -10 days (see Figure 2). This set ranges in rise time from 14 to 20 days in half-day intervals. The first derivative of the joined curves is not required to be continuous. In Section 4.3, we describe how we use the SDSS-II supernovae to determine which of these template curves is the best match to the observations.

Historically, there are a number of ways to characterize the light curve shapes of SNe Ia. For example, the MLCS2k2 $B$-band fiducial curve falls $1.1 \mathrm{mag}$ in 15 days after maximum, corresponding to a $\Delta m_{15}(B)=1.1 \mathrm{mag}$ (Hamuy et al. 1996b). Equivalently, the fall time, $t_{f}$, is defined as the number of days it takes a light curve to fade 1.1 mag after maximum brightness. The 2-stretch method estimates fall stretch, $s_{f}$, and the two parameters are simply related by $t_{f}=A \times s_{f}$, where $A$ is a constant that describes how long it takes the fiducial to fall $1.1 \mathrm{mag}$. The relation between $\Delta m_{15}(B)$ and $t_{f}$ depends on the precise shape of the fiducial curve, but is approximately quadratic for MLCS2k2:

$t_{f}=14.711-9.631\left(\Delta m_{15}(B)-1.1\right)+9.391\left(\Delta m_{15}(B)-1.1\right)^{2}$

over $0.7<\Delta m_{15}(B)<1.5$ for the MLCS2k2 fiducial $B$ band used here. We derived this equation by phase-stretching the MLCS2k2 fiducial light curve and directly measuring the $t_{f}$ and $\Delta m_{15}(B)$. Note that we use the literal definition of $\Delta m_{15}(B)$ and this may not be the same value derived by $\Delta m_{15}(B)$ fitters (Prieto et al. 2006) precisely because rise and fall rates are not absolutely correlated.

In order to estimate the rise and fall times of each supernova, an IDL $^{21}$ routine was developed that modifies the fiducial curve,

\footnotetext{
21 Interactive Data Language; http://www.ittvis.com/idl/idl7.asp
} 


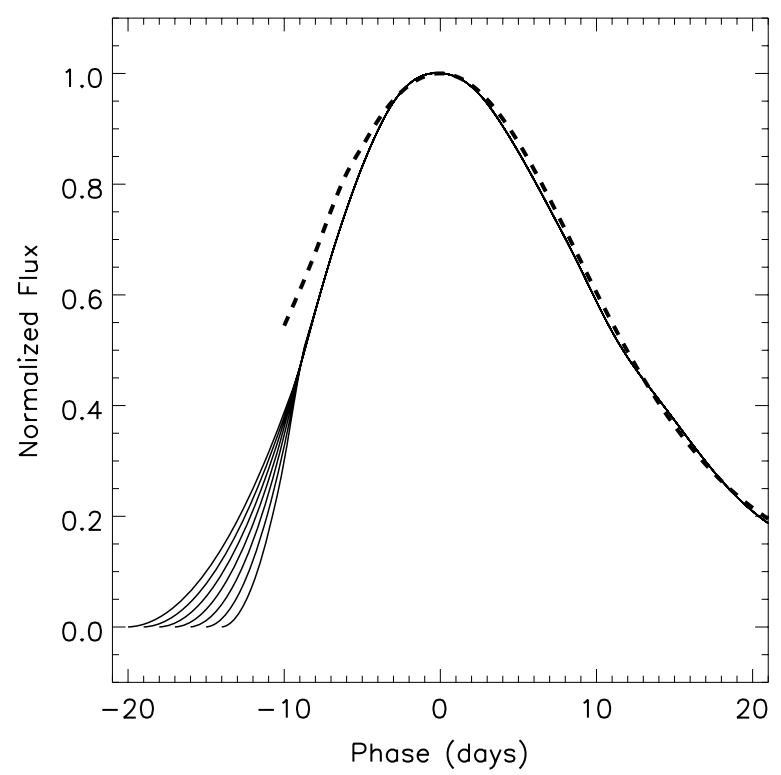

Figure 2. Comparison of the MLCS2K2 fiducial curves (solid), with the Leibundgut template (dashed) used by Riess et al. (1999). The difference between the Leibundgut template and our fiducial curve is 1.8 days at about $0.66 \mathrm{mag}$ on the rising portion, which is important in explaining the approximately 2 day difference between our rise time and that of Riess et al. (1999). Also, application of the single-stretch method using a very wide template will "squeeze" the light curve and push the maximum to a later date in order to fit the real rise data. The extrapolations shown here are described in Section 4.3; this figure displays 7 of our 13 extrapolations to the MLCS2k2 template.

$F(\tau)$, to fit the data at rest-frame times, $t$, by performing a $\chi^{2}$ minimization. Mathematically, the 2 -stretch function $S$ is defined as

$$
S(t)=\left\{\begin{array}{ll}
f_{0} F\left(\left(t-t_{0}\right) / s_{r}\right) & \text { if } t \leqslant t_{0} \\
f_{0} F\left(\left(t-t_{0}\right) / s_{f}\right) & \text { if } t>t_{0}
\end{array},\right.
$$

where $s_{r}$ is the rise stretch, $s_{f}$ is the fall stretch, $t_{0}$ is the time of maximum light, and $f_{0}$ is the peak flux. The fiducial curve is prepared so peak flux occurs at $\tau=0$ and the peak has a value of unity. The function $S$ is an approximation to the true observed light curve and is then interpolated at each observation time. The minimization is performed on data out to 25 days past maximum light. We applied the 2-stretch fitter to the SNe Ia studied in Riess et al. (1999) and a subsample from Hicken et al. (2009). In all, we fit 41 nearby $\mathrm{SNe}$ with the 2-stretch algorithm; this sample includes the eight SNe Ia analyzed by Strovink (2007), four more SNe Ia analyzed by Riess et al. (1999) that are not in the Strovink (2007) set, and 29 SNe Ia from CfA (Hicken et al. 2009) that had pre-maximum data. A number of SNe are common to all three data sets, and in these cases we use the data with the highest number of pre-maximum observations. SN 1996bo from Riess et al. (1999) is poorly constrained on the rise portion of the light curve, and was not included in our analysis. Our $\chi^{2}$ minimizing function was unable to find a suitable minimum for SN 1997bq and SN 1998ef from Riess et al. (1999). Lastly, SN 2005hk was not used from the CfA set because it is a peculiar SN Ia, and has been omitted from the SDSS-II analysis as well.

\section{METHOD}

This section will explain issues not fundamental to the 2-stretch fitter itself, but are important issues post-fit in analyzing the data. First, we discuss our error calculation method, and then we explain how we use the errors to select our subsample of the "best" SN Ia light curves. Next, we describe our method for template selection, ultimately resulting in the selection of a 16.5 day rise-time MLCS2k2 template which is used for all 2-stretch fits in this paper. Lastly, we discuss how we combine the $B$ - and $V$-band fits for each SN Ia.

\subsection{Method of Estimating Errors}

The uncertainty in rise and fall measurements arises from both photometric errors and the distribution of observations across the light curve. To estimate the rise/fall error, we use a Monte Carlo method similar to that of Contardo et al. (2000). At each observation in a light curve, we employ a Gaussian distribution centered on the observed flux and with a standard deviation equal to the flux error of that point. We generate simulated photometry at each observed time and then perform the 2-stretch $\chi^{2}$ minimization on the simulated light curve. The randomization is performed 100 times, and the standard deviation of the set is used as the error for the rise/fall times. There is no distinguishable difference in error values if this is performed 100 times or 1000 times. The rise/fall-time uncertainties reported by this method are used as a measure of the quality of the fit of each SN.

For the low-redshift supernovae, we found that the photometric uncertainties reported in the published light curves had a large range even though the supernova apparent peak brightnesses were similar. We assume this is due to some authors including systematic errors (photometric calibration uncertainties) in some analyses while other studies list only statistical photometric uncertainties. We add 0.01 in quadrature to all error values to take into account uncertainty in the model.

We notice a bias toward larger errors on fast-declining supernovae. The reason for this is twofold: these supernovae are generally fainter for a given redshift and therefore have larger photometric errors, and the rest-frame cadences of observations of these supernovae are longer. By excluding supernovae with large rise/fall error estimates, we have reduced the number of very fast decliners in our sample relative to supernovae with normal and slow-declining light curves. By a similar argument, we expect that the number of broad light curves is overrepresented in this sample when compared to the number of fast-declining events.

\subsection{Selecting Well-sampled Light Curves}

To determine how the rise and fall times of individual $\mathrm{SNe}$ Ia are related, we had to choose supernovae with well-defined light curves before and after maximum light. Sorting supernovae based on the number of points within a certain time range does not take into account the variation in photometric quality at various redshifts. We decided to select light curves based on the rise- and fall-time errors calculated by our Monte Carlo method.

Since one of our goals was to look for the double-peaked RMF distribution found by Strovink (2007), which has a separation of about 3 days, we decided to use a 2.0 day error cut on the rise time and the fall time. We applied these cuts to both the $B$ and $V$ bands before averaging the stretches from each band. These criteria produce a sample of 105 supernovae, out of the 391 SDSS-II SNe. The redshifts for these events range between 0.037 and 0.230 ; the average redshift is 0.14 .

Just as in the single-stretch method, it is possible to "correct" the observed light curve to best match the fiducial curve by dividing the time axis by the estimated stretch parameter. This 


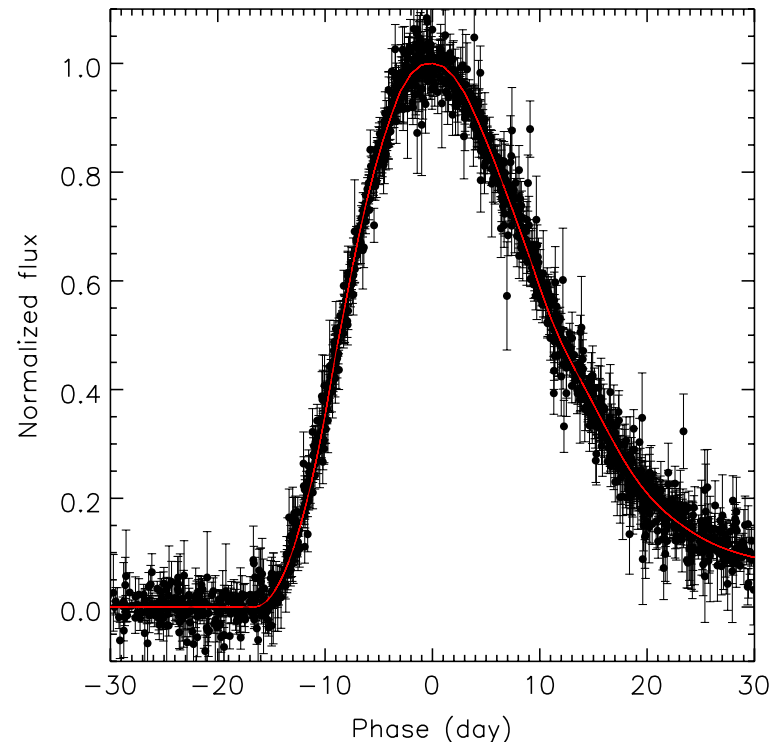

Figure 3. The $105 \mathrm{SNe}$ used in this study, corrected by both the rise and fall stretches, along with the flux and time of maximum found by the fitter using a 16.5 day rise-time template. The rise time of the best template is not the average rise time of the data, but a measure of the best relative shape between the extrapolated early rise portion and the MLCS2k2 template. This represents $B$ band only. This normalized light curve has a $\chi^{2} /$ dof of $211 / 247$, a probability of fit of 0.988 . This fit is much better than the single-stretch version, which has a probability of fit of 0.242 .

(A color version of this figure is available in the online journal.)

normalizes the light curve to match the template curve that was used in the fitting process. The only difference for the 2-stretch method is that the pre-maximum and post-maximum time axes have different stretches applied. Figure 3 shows the 2-stretchcorrected light curves for the 105 SDSS-II light curves well sampled in both rise and fall.

\subsection{Selecting the Fiducial Curve}

The MLCS2k2 fiducial light curves were poorly defined for early phases, so we constructed a set of extrapolated curves with a range of explosion dates that meet the MLCS2k2 curve at -10 days. Recall that in the simple fireball model, the extrapolations are quadratic functions with zero flux at the day of explosion. We created extrapolations at half-day intervals starting with explosion at 20 days before $B$ maximum and ending with explosions at 14 days before peak (see Figure 2).

To determine the best extrapolation, we fit each of the 105 light curves with the 2-stretch algorithm using each of the 13 extrapolated rise curves as a fiducial. We then divide the preand post-maximum portions of each light curve by the derived rise and fall stretches, and combine all 105 light curves into a single normalized light curve for each candidate fiducial curve. We calculate a $\chi^{2}$ value on the data points contained within the region of phase from -20 to -10 days. We found that the smallest reduced $\chi^{2}$ value and largest corresponding probability of fit were attained with the fiducial curve with a 16.5 day rise time in the $B$ band (see Figure 4). This template was best in $V$ band as well. This MLCS2k2 16.5 day template is the template that was used for all analyses in this paper. We emphasize that this number is not our estimate for the typical rise time of a Type Ia $\mathrm{SN}$, but a determination of the shape of the early light curve that best matches the data, relative to the shape of the actual MLCS2k2 template data later than -10 days. We also applied a single-stretch fit and found that the probability of fit was always

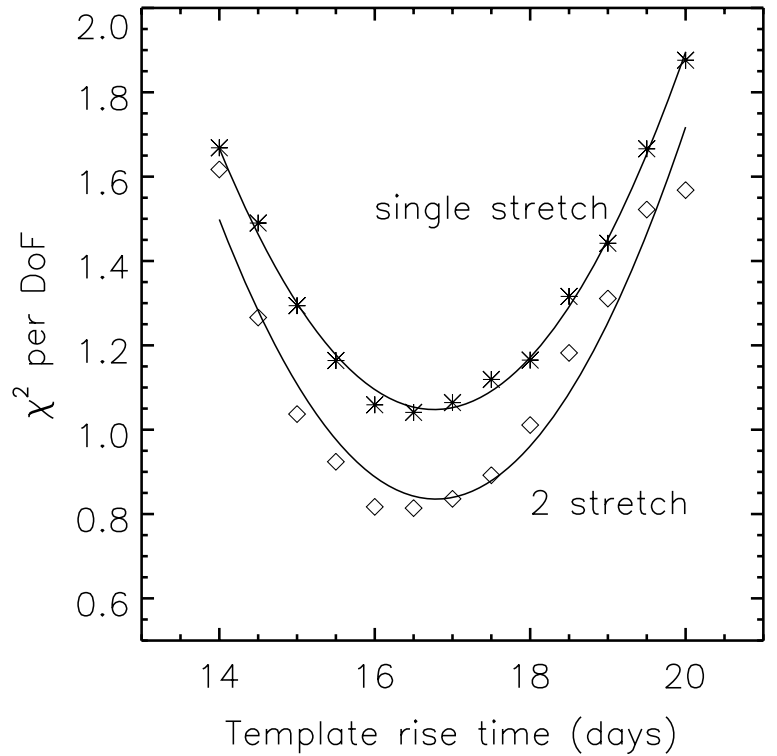

Figure 4. Plot of the $\chi^{2}$ distribution for the fiducial curve rise times, consisting of 105 high-quality SDSS-II light curves. The light curves were normalized to the fiducial curve using the values determined by our 2-stretch fitter, then the reduced $\chi^{2}$ value of the region between -20 and -10 days was calculated. Plotting the reduced $\chi^{2}$ for the entire light curve fitting region produces a similar result in the comparison of single stretch vs. two stretch, but is slightly noisier. This data represent the $B$ band only.

significantly smaller than for the 2-stretch fit, showing that the 2 -stretch fitting method is a better model for SN Ia light curves (see Figure 4). The $\chi^{2}$ value goes from 1515 (single stretch) to 1140 (2-stretch) with the addition of another model parameter, with 1050 and 951 degrees of freedom (dof), respectively. At the minimum $\chi^{2}$, the equivalent probability of fit for the 2-stretch fitter is 0.988 while for the single-stretch fitter it is 0.242 . Lastly, we used our error calculations to determine the optimum day after maximum to cut off the fitting algorithm. The fraction of the 391 supernovae that pass our error cuts is a good estimator of the overall quality of the fits. We fit all of the light curves with a range of cutoff ages from +15 days to +35 days and found the best cutoff to be around +25 days. Longer age cutoffs can bias the fall time with noisier data that may not be as well represented by the template; we found that the best combination of small errors and negligible bias occurred at the cutoff age of +25 days. This age cutoff was used in all analyses reported in this paper.

\subsection{Combining the B- and V-band Stretches}

Following Strovink (2007), the stretches from the $B$ and $V$ bands are combined to produce a single rise and a single fall-time estimate for each supernova. The stretches in the two bands $\left(s_{r}(B)\right.$ and $s_{r}(V)$, for example), found using the 16.5 day MLCS2k2 template to find the stretches, are combined using their weighted average, with our derived errors as the weights (in the form $1 / \sigma^{2}$ ). This has the advantage of reducing noise in the rise/fall-time measurements. Figure 1 shows that the SDSS-II $B$-band light curves, on average, decline more slowly than the MLCS2k2 fiducial. This could be due to selection bias that comes from the tendency of a magnitudelimited search to find brighter than average events that also tend to have slower-declining light curves. However, the average SDSS-II $V$-band light curve is a good match to the MLCS2k2 $V$-band fiducial. This mismatch between the average $B$ and 

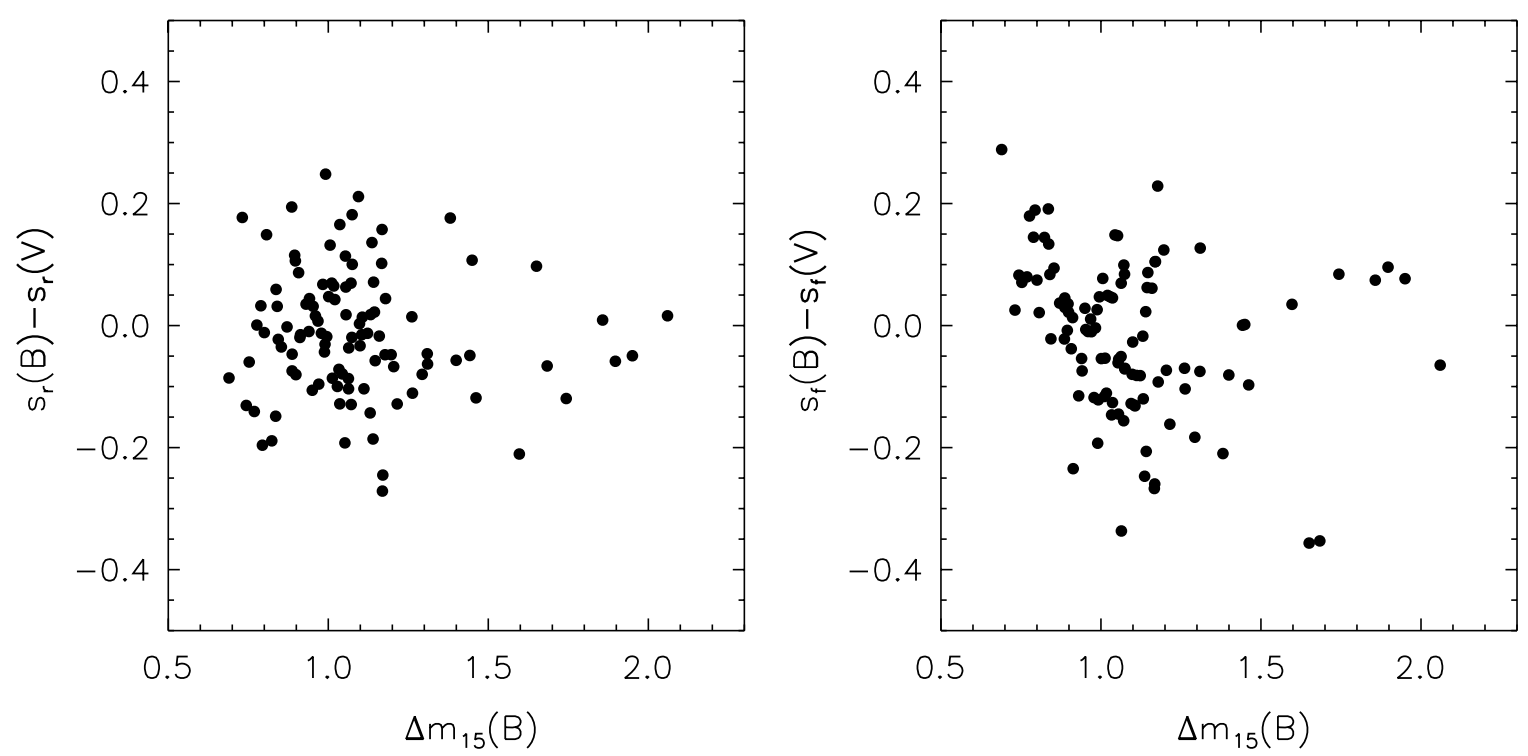

Figure 5. Left: the $B$-band rise stretch minus $V$-band rise stretch for 105 SDSS-II Type Ia SNe. The selection criterion for these 105 is described in Section 4.2 . The $B$ - and $V$-band rise stretches show no clear variation across the sample, indicating that the rise stretch is consistent between the two bands. Right: the difference between the $B$-band fall stretch and the $V$-band fall stretch for the same $105 \mathrm{SNe}$. Here, the data show evidence for variation with $\Delta m_{15}(B)$. Specifically, we find that slow-declining events have a larger than expected $B$-stretch than $V$-stretch when compared to events with $\Delta m_{15}(B)>1.1$.

$V$ curves and the fiducial is puzzling. We have chosen to ignore this difference and simply renormalize the fiducial stretches to best match the SDSS-II average light curves in both bands.

We found that the MLCS2K2 templates in $B$ and $V$ were different between their average rise and fall stretches when compared to the SDSS-II data. The $V$-band stretches on the fall portion were on average smaller than the $B$ band, while on the rise portion the $V$-band stretches were larger than $B$ band. In order to renormalize our template, we used the average stretches from the set of 105 SDSS-II SNe. We divide all $B$ - and $V$-band stretches of the actual data by the average $B$-rise, $B$-fall, $V$ rise, and $V$-fall stretches. This ensures that the templates have the same rise and fall stretches in both $B$ and $V$, so that we can average the stretches together with confidence that they are centered about the same mean value. This method of modifying the stretches of the data after fitting relies on the fact that starting our $\chi^{2}$ minimizer at a different initial condition will still provide the same final result. The other choice is to modify the template before fitting, in which case we would be multiplying the template by these average stretches from our data set. As long as the $\chi^{2}$ minimizer finds the same minimum, these two methods are interchangeable. Testing indicated this to be the case.

To clarify the relation between $B$ and $V$ light curves, the difference between the rise stretches and fall stretches after renormalizing the templates for the two bands is plotted in Figure 5. We find that on the rise, the $B$ - and $V$-band stretch difference is constant with decline rate, suggesting that the shape of the rise portion of the fiducial curve is consistent between the two wavelengths. But the plot of $s_{f}(B)-s_{f}(V)$ shows a kink near $\Delta m_{15}(B)=0.9$, indicating that the fading portions of the $B$ and $V$ light curves require two different stretch parameters to match the data. Specifically, very slow declining events tend to fade more slowly in the blue than in the visual band when compared to normal decliners. This effect should make very slow decliners more blue after maximum than their faster-fading cousins.

Clearly, the $B$-band fall stretch is not strictly correlated with the $V$-band fall stretch and this may indicate a physical difference between the slowest fading events and more normal-declining supernovae. However, the effect is small enough that we do average the derived stretches for this analysis.

\section{ANALYSIS}

\subsection{Rise versus Fall Times in the SDSS-II}

Using our sample of 105 high-quality SDSS-II supernovae, with the 16.5 day MLCS2k2 template for the fits, we obtain an RMF distribution that is best described by a single Gaussian distribution. This distribution can be seen in Figure 6, which shows a standard histogram of the RMF distribution along with an ideogram of the distribution. ${ }^{22}$ These histograms, along with the actual rise and fall distributions shown in Figure 7, are suggestive of a broad, single-peaked distribution in RMF for the SDSS-II supernovae. The width of the RMF histogram is wide compared to the errors in the measured rise and fall times. This implies that the rise time is not strongly correlated with the fall.

The ideogram in the right panel of Figure 6 is a visual device only and is used to display a histogram of the data weighted by errors in $t_{r}-t_{f}$. It is not used to numerically evaluate any properties of the data. The distribution of a standard histogram, one where the data values are separated into rigid bins and then simply counted, can be very dependent upon the location and width of the bins. The ideogram, however, treats each data point as a Gaussian distributed about the mean value, so that the appearance of the distribution is only dependent upon the data values and their errors. For our ideogram, we weight the area that each RMF value contributes to the overall distribution by $1 / \sigma$. In other words, instead of normalizing the Gaussian distribution to 1 , we normalize to $1 / \sigma$. For each supernova RMF value, this gives us the distribution:

$$
D_{i}(x)=\frac{1}{\sigma_{i}^{2} \sqrt{2 \pi}} e^{-\frac{\left(x-\mathrm{RMF}_{i}\right)^{2}}{2 \sigma_{i}^{2}}},
$$

\footnotetext{
${ }^{22}$ Particle Data Group; http://pdg.lbl.gov/2007/reviews/ textrpp.pdf
} 

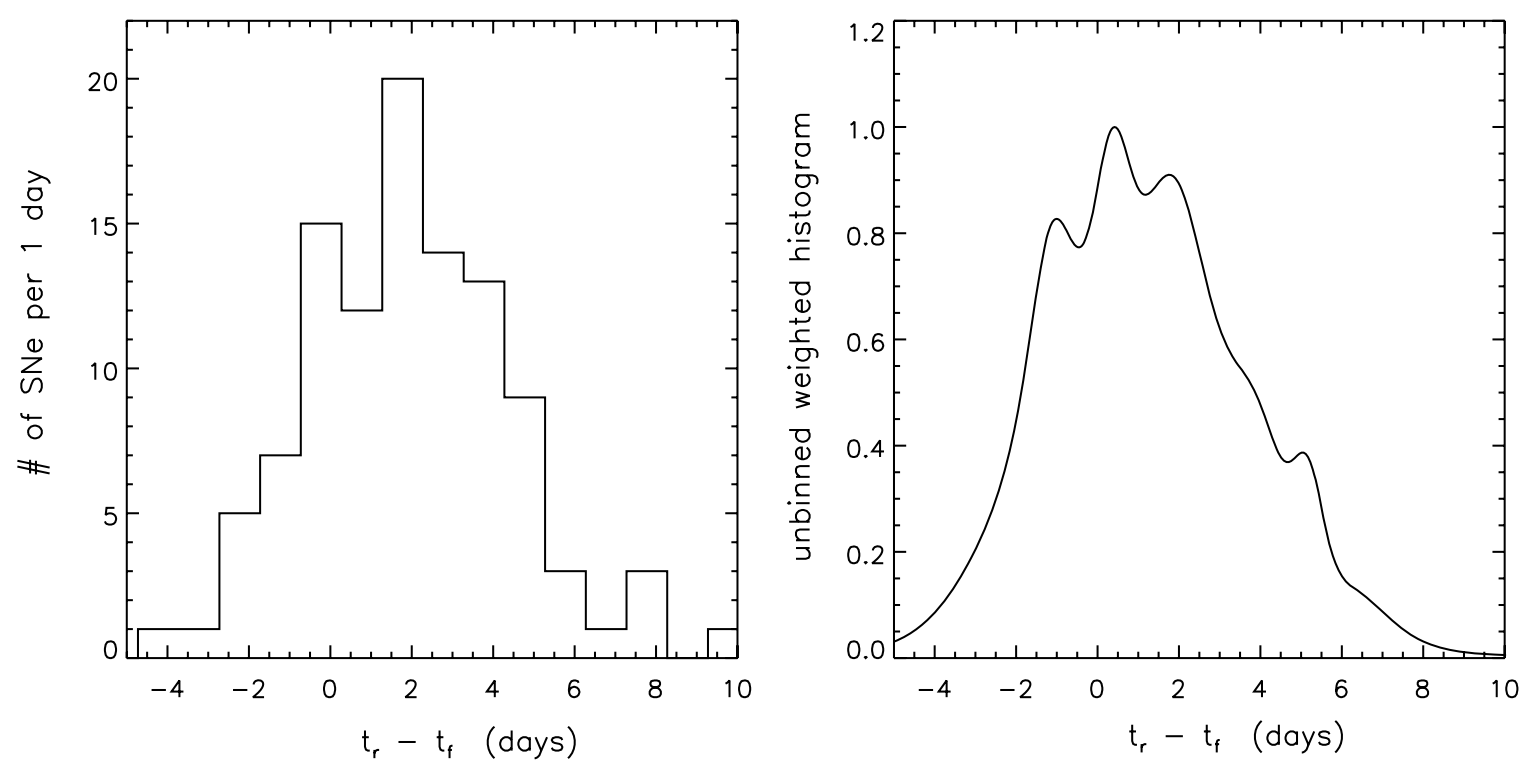

Figure 6. Left: standard histogram of the rest-frame $t_{r}-t_{f}$ distribution for 105 SDSS-II SNe. This figure is suggestive of only a single class of light curves, at least in terms of their $t_{r}-t_{f}$ values. Right: the ideogram of $t_{r}-t_{f}$ values, where each supernova is treated as a Gaussian and then these Gaussians are added together. This is also representative of a single distribution in $t_{r}-t_{f}$, and agrees very well with the standard histogram. The ideogram is described in detail in Section 5.1.
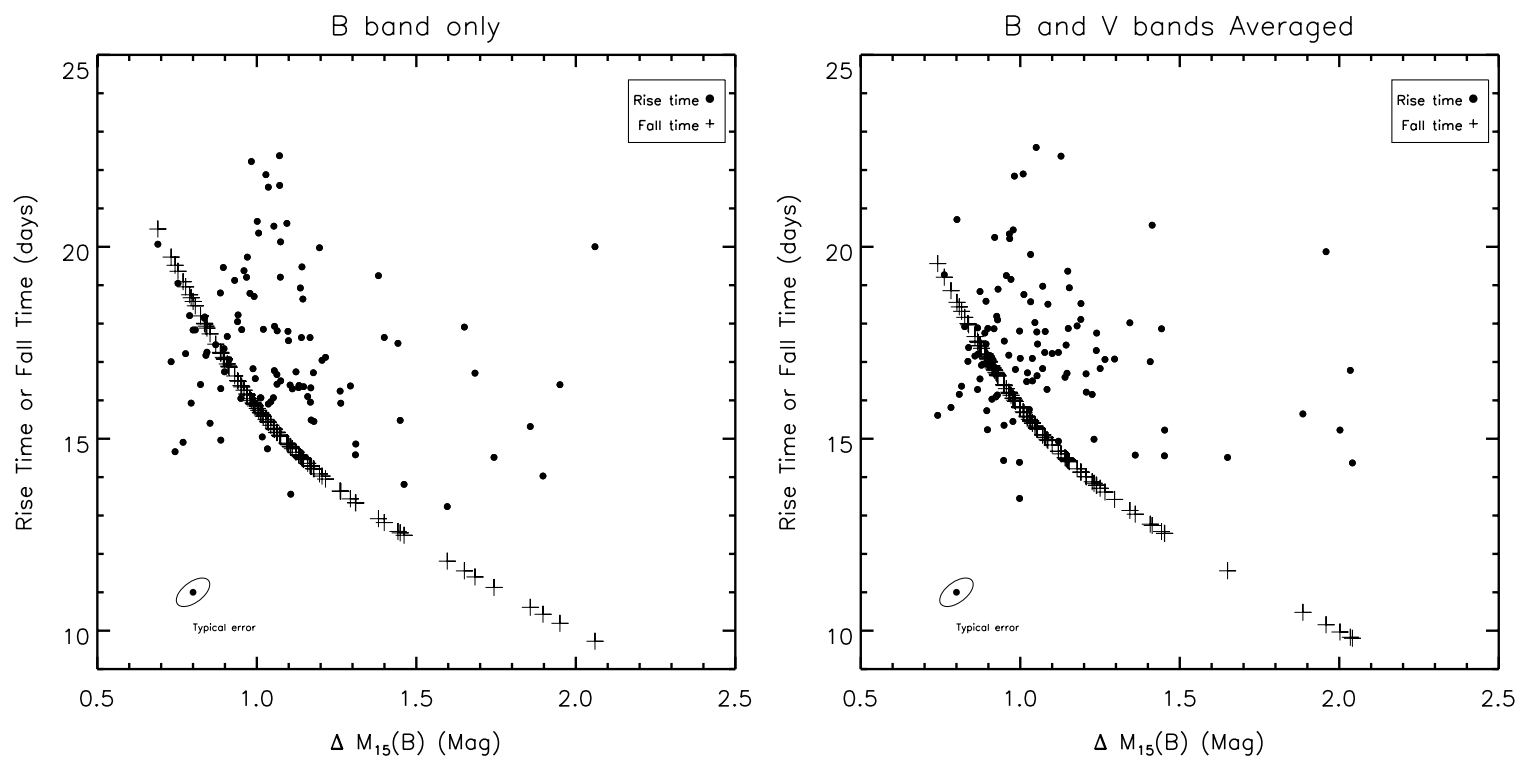

Figure 7. Left: the rise (dots) and fall times (crosses) vs. $\Delta m_{15}(B)$ for the 105 SDSS-II supernovae. Fall times are directly related to $\Delta m_{15}(B)$ and the crosses show the relation. These data are for $B$ band only, and are shown to reinforce that averaging the stretches introduces no bias to the data. The typical error ellipse for the rise time or fall time is shown in the lower right. The ellipse results from the covariance of the rise and fall estimates, resulting from the error in measuring the time of maximum brightness. Right: here, the $B$ - and $V$-band stretch estimates have been combined. Of special interest in this figure is the tendency for the slowest declining SNe to be among the fastest risers; however, there are very few slow-declining slow-rising SNe found in the sample. Also of note is a minimum rise time of about 13.5 days.

where $\sigma_{i}$ is the error in $\mathrm{RMF}_{i}$. The ideogram is then simply given by $I(x)=\sum_{i=1}^{N} D_{i}(x)$.

The $t_{r}-t_{f}$ values are not uniformly distributed with fall time $\left(\Delta m_{15}(B)\right)$. Figure 8 displays the RMF versus $\Delta m_{15}(B)$ for the 105 supernovae. We see that there are few slowly declining light curves $\left(\Delta m_{15}(B)<1.0 \mathrm{mag}\right)$ with large RMF values. In other words, many of the slowest declining SNe are among the fastest risers. This point is reiterated by the actual distribution of rise and fall shown in Figure 7.

For comparison with the SDSS-II data, we plot in Figure 8 the RMF trajectory of a 19.5 day rise-time fiducial curve with a single stretch applied to both rise and fall. This is currently the "standard model" of a SN Ia light curve and it does pass through much of the real data; however, the standard model cannot match the fast rising or very slow rising light curves in the SDSS-II sample.

\subsection{Average Rise Time and Decline-rate-corrected Curves}

Using our errors to weight the rise times of the sample, we obtain for the nearby set of SNe Ia an average rise time of $16.82 \pm 0.28$ days (standard error) with a standard deviation of 1.77 days. For the SDSS-II SNe Ia, we obtain a weighted average rise time of $17.38 \pm 0.17$ days (standard error) with a standard deviation of 1.8 days. These values are in general agreement, especially considering that the SDSS-II supernovae and the local sample have different selection biases. This result 


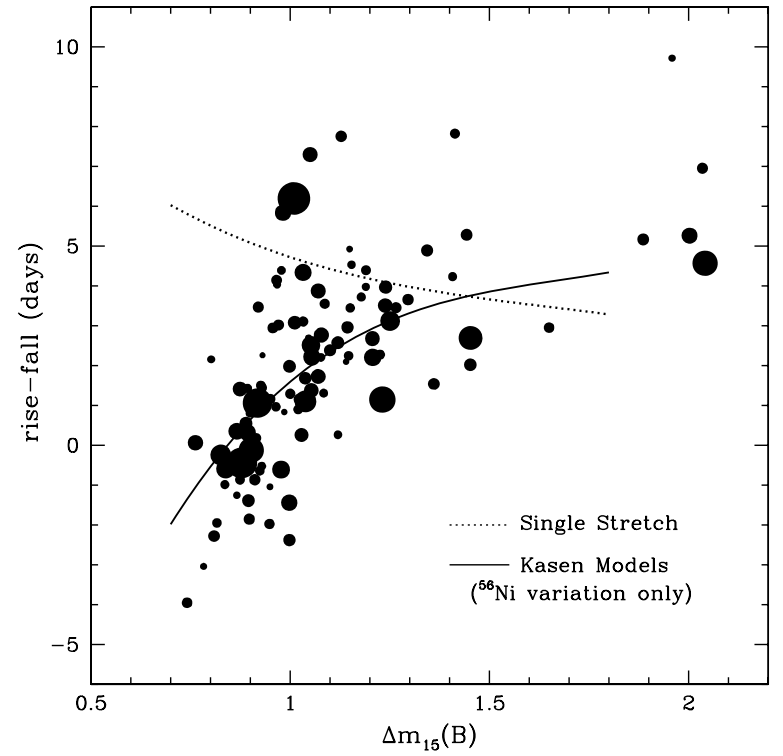

Figure 8. Plot of $t_{r}-t_{f}$ against $\Delta m_{15}(B)$, showing only the 105 SDSS-II supernovae. This figure displays the unexpected result that the slowest declining $\mathrm{SNe}$ tend to be the fastest risers. The dotted line shows $t_{r}-t_{f}$ vs. $\Delta m_{15}(B)$ relation if $\mathrm{SNe}$ Ia followed a single-stretch model with a rise time of 19.5 days and a fall of 15 days. The solid line shows the predicted $t_{r}-t_{f}$ for a series of Kasen light curve models that vary total radioactive nickel yield. The size of the points represents the error, so that larger points have smaller error.

agrees very well with that of Strovink (2007), who found an average rise time of $17.44 \pm 0.39$ days in the nearby sample.

In order to gain further insight into the SDSS-II light curves, it is beneficial to perform a decline-rate correction to the sample of light curves, similar to the procedure described by Strovink (2007). This correction is performed by dividing the entire time axis of each SN by the fall stretch found by the 2-stretch fitter. Based on the ideas of the single-stretch method, that every SN Ia light curve maintains the same relative shape between rise and fall, this approach should normalize the entire light curve to the shape of our template.

We applied a decline-rate correction to the $105 \mathrm{SNe}$ in our data set, by dividing the entire time axis by $s_{f}$ only, and found that the spread of the rise portion of the light curves increased. For the SDSS-II SNe Ia, the average decline-rate-corrected rise time is $17.32 \pm 0.12$ with a standard deviation of 2.89 days. The increase in the spread of the rise times after applying the decline-rate correction is a departure from the expected result implied by a single-stretch method.

This increase in the rise-time spread can be seen graphically in Figure 9, which shows the result of applying the decline-rate correction to the $105 \mathrm{SNe}$ Ia that pass our error cuts. This plot uses $B$-band data only, along with $B$ band only stretches, not the weighted average that was used in the RMF analysis. The SNe plotted in red have RMF $\leqslant 2.0$ days, while the ones plotted in blue have RMF $>2.0$ days (the color selection for these supernovae is not meant to imply anything about the intrinsic colors). The plot on the left of Figure 9 contains the SNe with no stretch corrections. Even without stretch corrections, the data show the distinct light curve shape differences in $\mathrm{SNe}$ Ia that are also shown graphically in Figure 8. Without stretch correcting, we see clearly that most of the slowest declining supernovae are also the fastest rising supernovae; this effect becomes more apparent after decline-rate correction. The slow-declining, fastrising light curves (in red) rise even faster, while the faster declining, slower rising light curves (in blue) rise more slowly.
The dispersion in rise time increases from the uncorrected mean value, since the average rise times agree between the corrected and uncorrected distributions. This is more evidence that current SN Ia light curve fitting models are not able to acceptably characterize the full range of SN Ia light curve shapes. We have arbitrarily selected the dividing point at 2 days in $t_{r}-t_{f}$, which is the mean value, and we emphasize that the light curves show a continuum of $t_{r}-t_{f}$ values displaying minimal evidence for a two-group classification using this parameter.

\subsection{Comparing the 2-stretch Fitter with Single Stretch}

In order to further examine the result that the 2-stretch fitter is a better representation of SN Ia light curve shapes, we selected a subset of 99 SDSS-II SNe Ia (from the 105 that passed our 2-stretch only error cuts) that passed our error cuts using both the 2-stretch fitter and a single-stretch fitter, and calculated the $\chi^{2}$ value of the stretch-corrected light curve. In this analysis, we calculated $\chi^{2}$ over the entire region that was fit. For the 2 -stretch fitter, this composite light curve has a $\chi^{2}$ value of 1140 with 951 dof. For the single-stretch fitter, this composite light curve has a $\chi^{2}$ value of 1515 with 1050 dof. Adding another parameter to a fitting procedure should be expected to reduce the $\chi^{2}$ value by approximately 1 (for each individual fit, so in this case 99) if that fitting parameter does not add any new useful information to the results. In our case, the 2 -stretch fitter has a significant impact in reducing the $\chi^{2}$ value of the composite light curve, demonstrating that the 2 -stretch fitter is a better model for representing SN Ia light curves than a single-stretch fitter.

\subsection{Fitting Simulated Light Curves}

As part of the SDSS-II supernova analysis, a software package called SNANA was developed to analyze data and to create simulated light curves (Kessler et al. 2009b). SNANA can create a set of supernova light curves with the same cadence, redshift distribution, and photometric signal-to-noise ratio as the SDSS-II survey, created from a variety of light curve models. In this section, we test the MLCS2k2 model and the single-stretch model against the real SDSS-II SN Ia data.

We synthesized 2694 simulated light curves using a 17 day rise-time MLCS2K2 template with a range of $\Delta$ values and extinction that matches the analysis of SDSS-II SNe Ia done in Kessler et al. (2009a; see Section 6). These simulated curves have a range of $z$-values from 0.019 to 0.35 with an average of 0.19 . We applied the same error cuts to these simulated curves as we applied to the SDSS-II data; the $z$-values after error cutting range from 0.019 to 0.24 with an average of 0.12 . This simulated distribution after error cutting is shown in Figure 10. It displays characteristics that are present in the SDSS-II SNe as well; it contains many SNe that are very slow decliners but are among the fastest risers. However, the simulation is lacking SNe that have relatively normal fall times yet are slow risers such as SN 1990N.

We also synthesized 2651 curves using a 17 day rise-time template simulated with a single-stretch model. The stretch values are based on the same analysis from Kessler et al. (2009a). The $z$-values range from 0.019 to 0.35 with an average of 0.25 . After error cutting, the $z$-values range from 0.019 to 0.34 with an average of 0.18 . We fit these simulated curves with the 2 -stretch fitter, but their underlying distribution is based on single stretch. The results can be seen in Figure 10. As expected, this single-stretch simulation shows a strong correspondence 

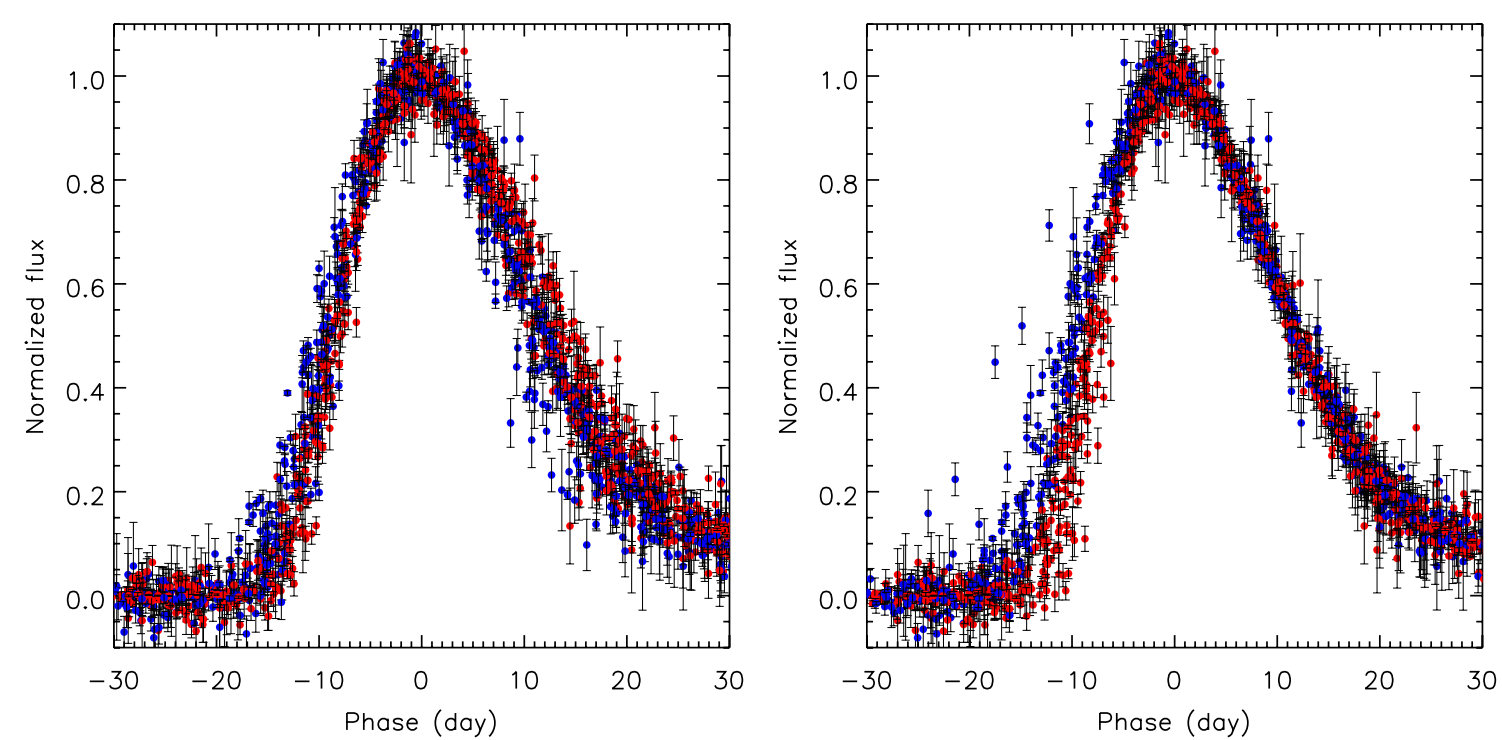

Figure 9. Left: the $105 \mathrm{SNe}$ Ia used in this study, with no stretch corrections applied and $B$-band data only. The red points represent SNe Ia with $t_{r}-t_{f} \leqslant 2.0$ days, while the blue points represent SNe Ia with $t_{r}-t_{f}>2.0$ days. Right: the same supernovae, with the entire time axis divided by the fall stretch as found by the 2-stretch fitter, showing the tendency for the slowest decliners to be the fastest risers. This analysis implies that the previously accepted correlation between rise and fall, e.g., a single-stretch model, is not supported by the data once the rise and fall times are disconnected in the fitting process.
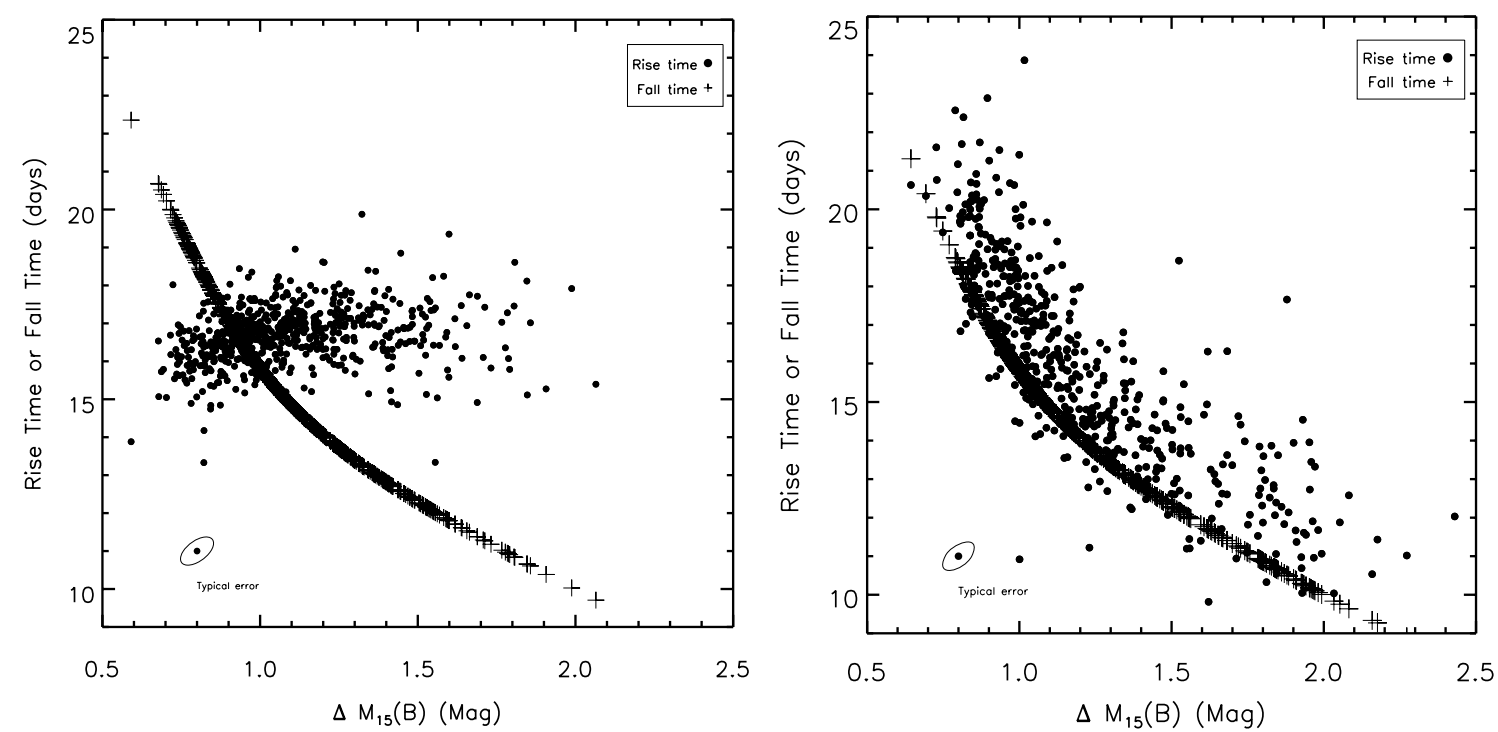

Figure 10. Left: distribution using SNANA simulated curves modeled with the MLCS2K2 17.0 day rise-time template. From an initial set of 2694 simulated light curves, 620 pass our error cuts, a very similar fraction as for the real data. Note that the MLCS2K2 model does not capture the true spread in rise times observed in the SDSS-II data. The rise time stays mostly constant, except in slowly declining SNe where it decreases with increasing fall time, an interesting correlation between rise and fall that is observed in the SDSS SNe as well. Right: simulated curves from the same input template but for a single-stretch model, and fit with the 2-stretch fitter. The rise time follows the fall time exactly for all values of decline rate, and there is no apparent trend for slow-declining light curves to have fast rise times in this single-stretch simulation.

between rise and fall that is not apparent in the SDSS-II data. Upon comparison with Figure 7, there is a noticeable difference in the spread of RMF values, with the SDSS-II data having a much greater range. Once again this implies a lack of correlation between rise and fall that is unachievable in this single-stretch model of light curve generation. However, the single-stretch simulation does produce some normal-declining events that have very slow rise times.

Our simulated curves using an MLCS2k2 model and a singlestretch model imply that the range of SN Ia light curves is not fully matched with current light curve fitters. MLCS2K2 models appear to be slightly better than single-stretch models as they capture some of the slow-declining fast-rising $\mathrm{SNe}$ Ia that are evident in the real data. Indeed we expect MLCS2K2 to be better simply because it is trained on real SNe. Also note that the MLCS2k2 synthesized curves maintain an approximately constant minimum for the rise time of fast-declining $\mathrm{SNe}$ at around 13-14 days, which appears in the SDSS-II SNe but could be a result of meager numbers of these SNe Ia. This could be an indication of a physical constraint on the physics of SN Ia explosions, as it seems that increasingly fast decliners do not correspond to increasingly fast risers.

\section{DISCUSSION}

\subsection{Comparison to Previous Rise-time Studies}

We obtain an average rise time for the SDSS-II SNe Ia of $17.38 \pm 0.17$ days (standard error of the mean); this is significantly different from the result obtained in Riess et al. (1999). For the entire nearby sample, we obtain an average 
rise time of $16.82 \pm 0.28$ days (standard error). The standard deviation in the rise time is 1.77 days. This is in general agreement with the value reported for the SDSS-II data. The reason that our results differ from the 19.5 days found by Riess et al. (1999) is the difference in shape of the fiducial curves used in the two studies, and the application of the single-stretch fitter in previous methods. Aldering et al. (2000) prove that even slight variations in the declining portion of the template can change the measured rise time by 2 or more days using a single-stretch method. Figure 2 shows that the "Leibundgut" template (Leibundgut 1989) used by Riess et al. (1999) differs greatly from the MLCS2K2 curves in the pre-maximum phase. The Leibundgut template is $0.66 \mathrm{mag}$ fainter than maximum light at -10 days while the MLCS2k2 fiducial curve reaches $0.66 \mathrm{mag}$ at -8.2 days. Such a broad template combined with a single-stretch fit tends to force the rise to be very slow and to push the estimated time of maximum later than the true peak brightness (see Figure 7 in Riess et al. 1999). The narrower MLCS2k2 fiducial combined with the 2-stretch fitter shows that the rise is faster than that found by several previous studies and that there is a wide range of observed rise times for a given fall time.

We created extrapolations to the Leibundgut template in the exact same manner as for the MLCS2k2 template, ranging in rise time from 14 to 20 days in half-day intervals. Using the template determination method described in Section 4.3, we found the best extrapolated Leibundgut template had a rise time of 19.5 days (note that this utilizes the 2-stretch fitter). However, after our error cutting, we obtain an average rise time of $17.18 \pm 0.19$ days using this template. Even though the extrapolated Leibundgut template has a longer rise time than the MLCS2k2 template, the stretches obtained are smaller so that the average is consistent. Indeed this should be expected with the 2-stretch fitter; the template with the best relationship between early rise and later rise is selected, but the overall rise time remains constant despite the difference in the fiducial relationship between rise and fall.

To further demonstrate this point, we also performed a risetime extrapolation using the same method as Riess et al. (1999), as well as using the Leibundgut template. We fit all 391 SDSS-II SNe Ia with a single-stretch fit using only data from -10 days to 25 days, and we cut SNe with stretch errors greater than 2.0 days. After stretch correcting the full light curve, we used the data less than -10 days (which was not used in the fitting process) to fit a parabolic extrapolation to explosion. Using this method, we obtain an extrapolation of 19.64 days for the SDSSII SNe Ia. This agrees quite well with both the results of Riess et al. (1999) and our own template selection using Leibundgut template extrapolations and the 2-stretch fitter.

We have shown in this section that the difference between our rise time and that of previous studies revolves around the flexibility of the 2-stretch fitter regarding the template used. A single-stretch fitter that is used to determine the best extrapolation to explosion is much too reliant on the relationship between rise and fall in the template. The 2-stretch fitter is more flexible in the sense that the input template can have any relationship between rise and fall and the average rise time will be consistent with other templates. Performing a singlestretch fit and extrapolating to explosion gives the same result as our template determination method from Section 4.3. However, in order to find the average rise time, the addition of another parameter to independently estimate the rise stretch is required, because the rise and fall are not strictly correlated.

\subsection{Shape of the Early Rise}

As with previous studies, we have assumed that the optical flux rises as $t^{2}$ soon after explosion. Arnett (1982) showed that adiabatic losses should nearly balance heating by radioactive decay and keep the effective temperature relatively constant. Thus, the luminosity goes as the radius squared and therefore the time squared for a constant expansion rate. Conley et al. (2006) directly tested this model by fitting light curves with the temporal power-law index as a free parameter. They found the temporal index $n$ that best matched the low- and highredshift supernovae was $1.8 \pm 0.2$, consistent with the Arnett calculation.

To estimate the shape of the early rise, we fit the 105 SDSS-II light curves with good rise and fall data with the 2-stretch fitter using only epochs later than -10 days. We then corrected all the light curves to the fiducial curve using the rise and fall stretches which provides $103 \mathrm{~B}$-band observations between -20 and -10 days before maximum. We then calculate the $\chi^{2}$ parameter over this interval by comparing the data to the function $f=A\left(t-t_{0}\right)^{n}$, where the time of explosion $\left(t_{0}\right)$ and power-law index $(n)$ are allowed to float. The value of the parameter $A$ is set by the condition that the function must meet the MLCS2k2 fiducial curve at -10 days.

We found the best-fit power-law index for the SDSS-II data was $n=1.80_{-0.18}^{+0.23}$. The index is strongly correlated with the time of explosion which we found to be $-16.8_{-0.6}^{+0.9}$ days. The result is consistent with the Arnett prediction and the Conley et al. (2006) estimate for the early light curve shape.

We can also test whether or not the early color of the SDSS-II supernovae follows Arnett's prediction of slow and modest temperature changes. For each SDSS-II observation where there is a good rest-frame $B$ and $V$ measurement, we construct a color based on the $V-B$ normalized flux difference. This essentially compares the supernova color to the color at $B$-band maximum and avoids the problem of dealing with magnitudes at low flux levels.

As a consistency check, for each supernova we take the flux color averaged between -15 and -10 days to see how it varies with redshift. We find that the color is essentially constant as a function of redshift which gives us confidence that the SNANA $k$-corrections at early times are self-consistent. We also see that the colors for $z>0.3$ are very noisy and we restrict our color analysis to the 332 events at redshifts less than 0.3 .

Figure 11 shows the flux color as a function of supernova phase. The colors have been binned by rest-frame day and the median calculated for each bin. As expected, the supernovae get significantly red after maximum as the $V$-band light curve fades more slowly than the $B$. Near the time of explosion, the color is slightly red and shifts to the blue just before maximum. This observed color change is exactly what is expected given the 2-stretch fit of the individual $B$ - and $V$-band light curves (and this is shown by the solid line in the figure). Using the median $B$-band flux, we convert the flux color into magnitudes and find the color index at -15 days is $B-V=0.5$ which shifts to $B-V=0.0$ at -9 days. The data earlier than -15 days is too noisy to attempt estimating a reliable magnitude color index. We convert the color index to an effective temperature using the empirical relation from Sekiguchi \& Fukugita (2000) and find that $T_{\text {eff }}$ rises linearly from $6000 \mathrm{~K}$ at -15 days to $9500 \mathrm{~K}$ at -9 days and then stays nearly constant until maximum.

This linear rise in temperature over the first week after explosion is not expected from Arnett (1982), and is puzzling. 


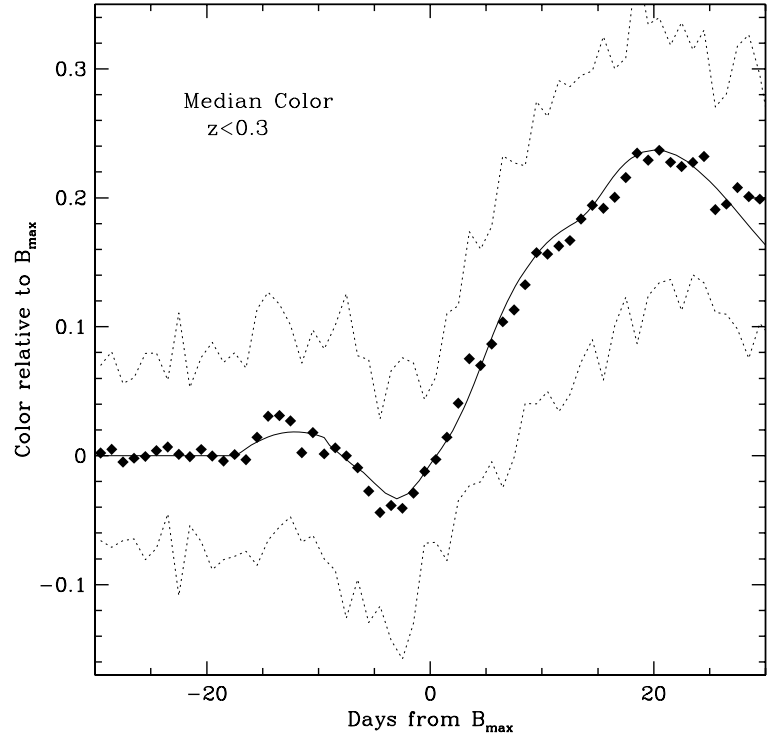

Figure 11. $V-B$ color relative to $B_{\max }$ as a function of $\mathrm{SN}$ phase. The data have been binned in 1 day (rest-frame) intervals and the median of each bin calculated. The diamonds represent the SDSS-II SNe with redshift less than 0.3 . The solid line represents the color of the template. The dotted line is the $1 \sigma$ spread of the data. Calculating a color index for times later than -15 days, we find that the effective temperature increases linearly from -15 to -9 days then remains nearly constant until maximum. This is not expected from Arnett (1982), which predicts that the effective temperature should remain constant. This implies that the power-law index should be nearer to $n \approx 4$, rather than the $n \approx 2$ calculated from the observations.

If the color variation represents a temperature change and the optical bands are following the bolometric flux, then we expect a temporal power-law index of at least $n=4$ instead of the observed $n \approx 2$. In conclusion, we find that the power-law index for the SDSS SNe Ia agrees with the Arnett prediction; however, we find that the early light curves do not follow the prediction of slow and modest temperature changes.

\subsection{Source of Rise-time Variation}

Our SDSS-II supernovae show a range of rise times for a fixed fall time, suggesting that the physics of the rise and fall epochs differ. Arnett (1982) and Pinto \& Eastman (2000a) show that the light curves of SNe Ia can be simply described by the deposition of energy from synthesized radioactive elements combined with the diffusion rate of energy out of the expanding nebula. Before maximum brightness the energy input rate from radioactivity exceeds the energy lost to luminosity. At maximum, the luminosity matches the instantaneous energy deposition rate and the decline from maximum correlates with the total radioactive yield. Using more detailed models, Woosley et al. (2009) show that the shape of the light curve depends on more than the radioactive yield. For example, increasing the production of intermediate mass elements (e.g., silicon and calcium) at a fixed nickel mass $\left({ }^{56} \mathrm{Ni}\right)$ narrows the light curve while increasing the peak luminosity. This occurs because the total burned mass correlates with the KE of explosion, and the faster the supernova expands, the earlier the radioactive energy diffuses out. In contrast, a low KE means a slow rise and faint maximum. Note that the variation in KE trends in the opposite direction to the Phillip's relation. Observationally, the radioactive yield and KE influence the rise and fall times in different ways and variation in these two parameters could help explain the range of rise times seen in the SDSS-II supernovae. Other explosion parameters, such as non-radioactive iron yield

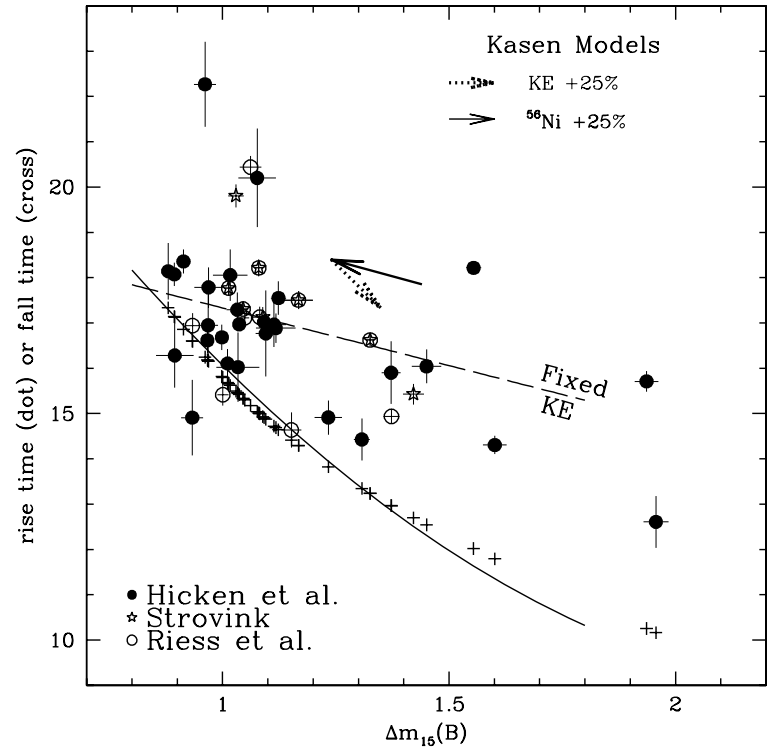

Figure 12. Plot of rise time and fall time vs. $\Delta m_{15}(B)$ for the 14 nearby SNe Ia analyzed in this study, with theoretical distributions derived from Kasen model light curves. The dotted line is a representation of the Kasen model rise times and its shallow slope implies that rise time is only mildly dependent on radioactive yield. The solid line is a quadratic fit to the fall times estimated from the models and are very consistent with the observed fall times. The arrows represent how a $25 \%$ change in $\mathrm{KE}$ or ${ }^{56} \mathrm{Ni}$ yield would change the position of a specific SN Ia from the Kasen models.

or degree of mixing, could also impact the rise versus fall times (Woosley et al. 2009). For illustration, we consider only KE variation here.

Kasen \& Woosley (2007) have calculated model light curves for $\mathrm{SNe}$ Ia with fixed $\mathrm{KE}$ and varying radioactive nickel masses to show that they can match the Phillip's relation fairly well. We can apply the 2-stretch method to these model curves almost as easily as to real data. From this we can see if the rise and fall times of the models can refine the physics of the observed light curve shapes. Unfortunately, the MLCS2k2 fiducial does not match the model light curves sufficiently well to estimate accurate rise and fall times. Instead, we chose the $M_{\mathrm{Ni}}=0.49 M_{\odot}$ model curve as a fiducial and applied the 2stretch fitter to the remaining models. The resulting rise times for fixed KE models with varying nickel yield are shown as a dashed line in Figure 12 and compared with observed lowredshift rise/fall times. The model rise time varies only modestly with $\Delta m_{15}(B)$ (slope of -2.6 days $/ \Delta m_{15}(B)$ ) compared to the fall time (slope of -9.2 days $/ \Delta m_{15}(B)$ at $\Delta m_{15}(B)=1.1$ ). This is in stark contrast to the single-stretch parameterization where the rise time parallels the fall time in this diagram.

We compare the predicted effect on rise/fall times from varying ${ }^{56} \mathrm{Ni}$ yield with the SDSS-II data in Figure 8. The observed trend of $t_{r}-t_{f}$ increasing with larger $\Delta m_{15}(B)$ is well matched by the model, although there are still many supernovae in the sample that lie far from the model. Clearly, the singlestretch parameterization of light curve shape is not justified by the observed rise/fall-time variations or predictions of the ${ }^{56} \mathrm{Ni}$ model. The small scatter in the rise portion of the SN Ia light curves seen in the full SDSS-II sample (Figure 1) should not be a surprise if it is a direct consequence of ${ }^{56} \mathrm{Ni}$ yield being the dominant source of diversity in SNe Ia (Arnett et al. 1985). This is a popular interpretation for variations in peak brightness in SNe Ia.

Kasen \& Woosley (2007) models that vary KE at fixed nickel yield were also fit with the 2-stretch method. Arrows in Figure 12 
illustrate the amplitude and direction in the rise/fall time versus $\Delta m_{15}(B)$ plane of a supernova that increases its nickel yield by $25 \%$ at fixed KE and then increases its KE by $25 \%$ at fixed radioactive yield. The change in KE produces a steeper variation in rise time than does nickel and in the opposite sense, i.e., increasing KE results in a faster light curve decay as well as a shorter rise time. Unlike nickel variations, changes in KE affect the rise time (slope of -9.7 days $/ \Delta m_{15}(B)$ ) as strongly as the fall time, meaning pure variations in KE result in almost no change in $t_{r}-t_{f}$ (and would be well fit by a single-stretch parameterization).

While this analysis focuses on the effects of $\mathrm{KE}$ and ${ }^{56} \mathrm{Ni}$ yield, there are other parameters that may be important to the light curve shape as well. In particular, mixing the ${ }^{56} \mathrm{Ni}$ to a larger radius may result in faster rise times (Pinto \& Eastman 2000b; Hoeflich et al. 2010). This effect plays a secondary role (Hoeflich et al. 2010) in light curve shape, however, and is not included in this analysis. The Kasen \& Woosley (2007) models used in this analysis contain no effects from variations in ${ }^{56} \mathrm{Ni}$ distribution. We have only employed model light curves with fixed iron mass yields, which is the parameter that controls the ${ }^{56} \mathrm{Ni}$ distribution in the one-dimensional Kasen \& Woosley (2007) models.

In principle, varying both ${ }^{56} \mathrm{Ni}$ yield and $\mathrm{KE}$ will allow a supernova to reach any point on the $t_{r}-t_{f}$ versus $\Delta m_{15}(B)$ diagram (Figure 8). Changes in radioactive yield will move supernovae diagonally, while KE variations shift supernovae horizontally. Given these motions, it is difficult to explain the handful of events with very slow rise times and normal decline rates $\left(t_{r}-t_{f}>6\right.$ and $\left.\Delta m_{15}(B) \approx 1.0\right)$. SN $1990 \mathrm{~N}$ may be a member of this group in the low-redshift set. The Kasen models also predict that peak luminosity should be related to nickel yield and $\mathrm{KE}$ variations, with an increase in ${ }^{56} \mathrm{Ni}$ and KE resulting in brighter events. Woosley et al. (2009) found, however, that the effect of these parameters on light curve width works in the opposite sense. An increase in KE narrows the light curve while increasing peak luminosity and a larger nickel mass widens the light curve while making a brighter peak. Examining the absolute magnitude estimates of the SDSS-II events may help sort out the origin of the rise/fall variations.

\subsection{Correlation with Host Galaxy Color}

Scannapieco \& Bildsten (2005) and Sullivan et al. (2006) have shown that rates of SNe Ia are strongly connected with host galaxy star formation rate. They model the rates as coming from two sources, one from a passive population of stars and the other a prompt population of supernovae correlated with high star formation rates. It is natural to speculate that the range of RMF corresponds to the sources of supernovae in the two stellar populations. To test this idea, we construct the $g-i$ color of the host galaxies of our sample of 105 SDSS-II supernovae. The $g-i$ color index correlates well with specific star formation rate measured from the SDSS spectroscopic sample. The host magnitudes are taken from the SDSS-DR5 catalog and are color corrected to the rest frame by interpolating from tables in Fukugita et al. (1996). The host galaxy color versus supernova $t_{r}-t_{f}$ is shown in Figure 13 for supernovae with $\Delta m_{15}(B)<1.5$ mag. Very fast declining supernovae are known to be associated with early-type galaxies (e.g., Gallagher et al. 2005; Hamuy et al. 1996a) and might confuse the result.

There is no significant correlation between host $g-i$ color and the rise-time properties of the supernovae. Supernovae with

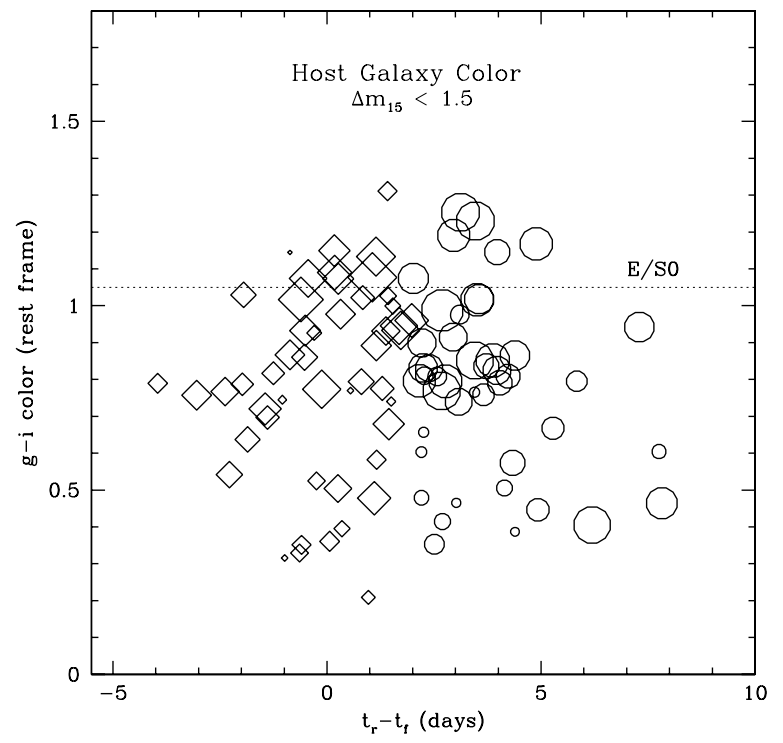

Figure 13. Host galaxy color vs. the difference in rise and fall times. Galaxies that hosted SNe Ia with $t_{r}-t_{f}<2.0$ days are plotted as diamonds while hosts with high RMF SNe are circles. The size of the plotting symbol correlates with the total brightness of the galaxy. The RMF cut at 2 days was selected arbitrarily. The host color distributions do not appear different between these two groups.

$\mathrm{RMF}<2.0$ days tend to have slower decline rates than the other supernovae and can be expected to result from the prompt, high star formation rate population. We do not observe this relation in our sample.

\subsection{Correlation with Hubble Residuals}

The Phillip's relation shows that the SN Ia light curve decline rate is related to the optical peak luminosity. The SDSS-II light curves suggest that there are a range of basic light curve shapes and this leads to several questions.

1. Which parameter is the best indicator of peak luminosity: fall time, rise time, total width, or rise minus fall time?

2. Is there a difference in the average peak luminosity between fast and slow risers for a fixed decline rate?

3. Do the color or dust properties vary with rise time?

To investigate these questions, we estimated the apparent peak $V$-band magnitude, and peak color by fitting the 105 light curves with good rise/fall-time measurements with MLCS2k2. We relaxed all priors to the fits (flatnegav) and assumed a fixed extinction law of $R_{V}=2.5$. Despite the good rise-time information in the light curve data, we restricted the fit to include only those points later than -10 days before maximum; this was done because we found rather poor fits to the light curves when using the MLCS2k2 template extrapolations that put the explosion date at -20 days before $B$ maximum. For this analysis, we cut nine supernovae with $\Delta m_{15}(B)>1.5$ because their colors are strongly dependent on decline rate (Garnavich et al. 2004).

We also fit the light curves using the SALT-II software (Guy et al. 2007) and compared the color parameter, $c$, and peak apparent magnitudes to those from MLCS $2 \mathrm{k} 2$. The average color difference was $(B-V)-c=-0.027$ with an rms dispersion of $0.045 \mathrm{mag}$ and the average apparent magnitude difference was $m_{V}$ (mlcs) $-m_{V}$ (salt) $=0.019$ with an $\mathrm{rms}$ dispersion of 0.038 mag. We conclude that for these wellsampled light curves the differences in fit parameters between MLCS2k2 and SALT-II are insignificant. 
Table 2

Hubble Residuals

\begin{tabular}{lccccccc}
\hline \multicolumn{1}{c}{ Fit } & $96 \mathrm{SNe}$ & $96 \mathrm{SNe}$ & $96 \mathrm{SNe}$ & $96 \mathrm{SNe}$ & $96 \mathrm{SNe}$ & $52 \mathrm{SNe}\left(t_{r}-t_{f}<2.0\right)$ & $44 \mathrm{SNe}\left(t_{r}-t_{f}>2.0\right)$ \\
\multicolumn{1}{c}{ Parameters } & 1 & 2 & 3 & 4 & 3 & 3 \\
\hline$M_{V}(\alpha)$ & -19.087 & -19.093 & -19.067 & -19.066 & -19.061 & -19.054 \\
$(B-V)(\beta)$ & $\ldots$ & -1.466 & -1.236 & -1.251 & -1.213 & -1.343 \\
$s_{f}-1(\gamma)$ & $\ldots$ & $\ldots$ & 0.614 & 0.583 & $\ldots$ & $\ldots$ & $\ldots$ \\
$s_{r}-1(\delta)$ & $\ldots$ & $\ldots$ & $\ldots$ & 0.792 & $\ldots$ & $\ldots .070$ \\
$s_{r}+s_{f}-2.0(\epsilon)$ & $\ldots$ & $\ldots$ & $\ldots$ & $\ldots$ & 0.691 & 0.600 \\
\hline$\sigma$ & 0.206 & 0.175 & 0.163 & 0.145 & 0.145 & 0.143 & 0.765 \\
$\chi^{2} /$ dof & $214 / 95$ & $147 / 94$ & $131 / 93$ & $100 / 92$ & $101 / 93$ & $59 / 49$ \\
\hline
\end{tabular}

Note. Residual $=m_{V}-5 \log \left(d_{L}\right)+25-\alpha+\beta(B-V)+\gamma\left(s_{f}-1\right)+\delta\left(s_{r}-1\right)+\epsilon\left(t_{r}+t_{f}\right)$.

Given the estimated apparent peak magnitude, redshift, $(B-V)$ color, and our measured rise/fall stretches, we minimized the residuals on the Hubble diagram calculated from

$$
\begin{aligned}
\Delta m= & m_{V}-5 \log \left(d_{L}\right)+25-\alpha+\beta(B-V) \\
& +\gamma\left(s_{f}-1\right)+\delta\left(s_{r}-1\right)+\epsilon\left(t_{r}-t_{f}\right),
\end{aligned}
$$

where $\alpha, \beta, \gamma, \delta$, and $\epsilon$ are coefficients allowed to vary in the minimization. The luminosity distance $\left(d_{L}\right.$ in $\left.\mathrm{Mpc}\right)$ is calculated from the known redshift and assuming the cosmological parameters of $H_{0}=65 \mathrm{~km} \mathrm{~s}^{-1} \mathrm{Mpc}^{-1}, \Omega_{\Lambda}=0.7$, and $\Omega_{m}=0.3$. Choosing a different set of cosmological parameters changes the relative absolute magnitudes by only a few percent at these redshifts. An "amoeba" algorithm was employed for the minimization of the $\chi^{2}$ parameter,

$$
\chi^{2}=\sum_{i} \Delta m_{i}^{2} / \delta \mu_{i}^{2},
$$

where $\delta \mu_{i}$ is the distance modulus uncertainty from the MLCS2k2 fit. We calculate $\chi^{2}$ for several combinations of the free parameters and the results of these fits are shown in Table 2.

The scatter about the Hubble line for a one parameter fit (zero point) applied to the remaining 96 events provides an rms scatter of $0.21 \mathrm{mag}$. This small scatter is not surprising given that the quality of the data is excellent and we have eliminated intrinsically red supernovae with the $\Delta m_{15}(B)>1.5$ cut.

Adding a color term reduces the scatter to $0.18 \mathrm{mag}$. The size of the color term, $\beta=-1.47$, should correspond to $R_{V}$, the ratio between $V$-band extinction and reddening in $B-V$, if dust were the source of the color variation. As noted for both local and high-redshift supernovae (e.g., Kessler et al. 2009a), the best-fit color term tends to be much smaller than the standard Milky Way extinction value of $R_{V}=3.1$. This may indicate non-standard dust in other galaxies or that the supernova colors are not well understood.

Minimizing the scatter by adding the fall stretch, $s_{f}$, as a third parameter has some effect on the Hubble residuals, reducing the scatter to $0.16 \mathrm{mag}$. A fourth parameter that includes the rise stretch, $s_{r}$, or the rise minus fall time, $t_{r}-t_{f}$, provides the smallest scatter of $0.145 \mathrm{mag}$. Surprisingly, the Hubble residuals are minimized when both the rise and fall stretches have large, positive coefficients. The Kasen models that vary both nickel yield and KE predict that increasing rise time (lowering KE) should reduce the supernova peak brightness, so the rise-time parameter would have the opposite sign from the fall-time parameter. Our hypothesis that KE has a strong influence on light curve shape and brightness does not match the observations well, but other variables should be explored. Varying the mass of stable iron elements appears to affect the rise time and brightness

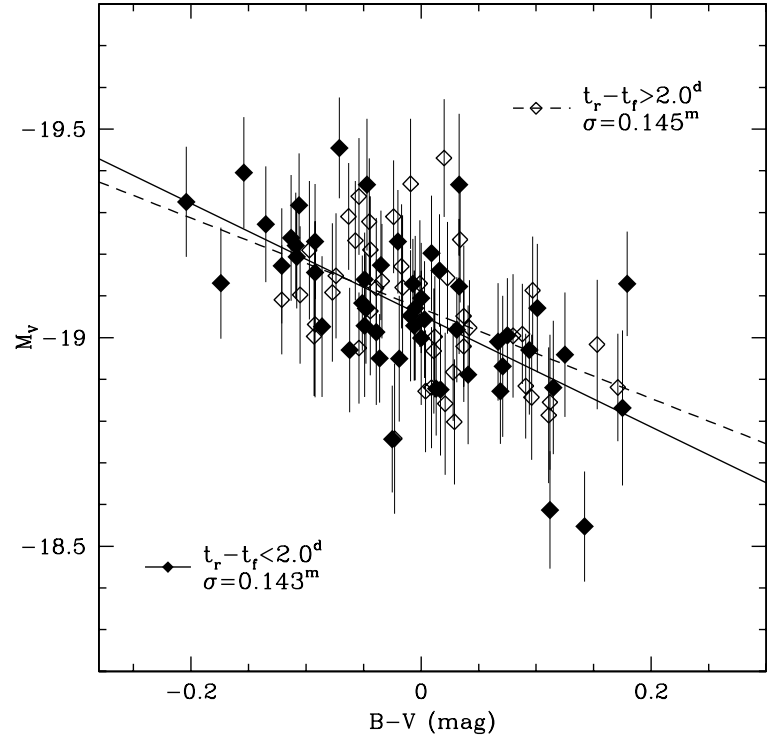

Figure 14. Absolute $V$-band magnitude vs. peak color for fast-rising $\left(t_{r}-t_{f}<\right.$ 2.0 days; filled diamonds) and slow-rising $\left(t_{r}-t_{f}>2.0\right.$ days; open diamonds) SDSS-II supernovae after correction for light curve shape. No difference in peak luminosity or color slope is apparent. Note, however, that the fast-rising events tend to be more blue at peak with 9 of the bluest 10 supernovae having $t_{r}-t_{f}<2.0$

in the same directions as the KE variation so are also disfavored by this analysis.

Applying a three-parameter minimization with the light curve characterized by $t_{r}+t_{f}$ results in the same scatter as a fourparameter fit with $t_{r}$ and $t_{f}$ as separate light curve shape indicators. This result suggests that it is the total width of the light curve that correlates with peak luminosity and not the rise or fall separately. Indeed, a three-parameter fit with $t_{r}-t_{f}$ does no better in reducing the scatter than a two-parameter minimization with just zero point and color.

When we divide the sample into a "low RMF" group with $t_{r}-t_{f}<2.0$ days and a "high RMF" subset with $t_{r}-t_{f}>$ 2.0 days there appears to be no substantial differences in the Hubble residuals. Applying a three-parameter minimization (zero point, color, and total stretch), we find the color term for the low and high RMF is only marginally different (see Figure 14). We note, however, that the low RMF group has more blue supernovae than the high RMF group, with nine of the 10 bluest events having $t_{r}-t_{f}<2.0$ days. The relation between luminosity and full width of the light curve also shows a little difference between the two rise-time divisions.

The average absolute magnitudes of the two groups after color and light curve shape correction differ by less than $0.05 \mathrm{mag}$, 


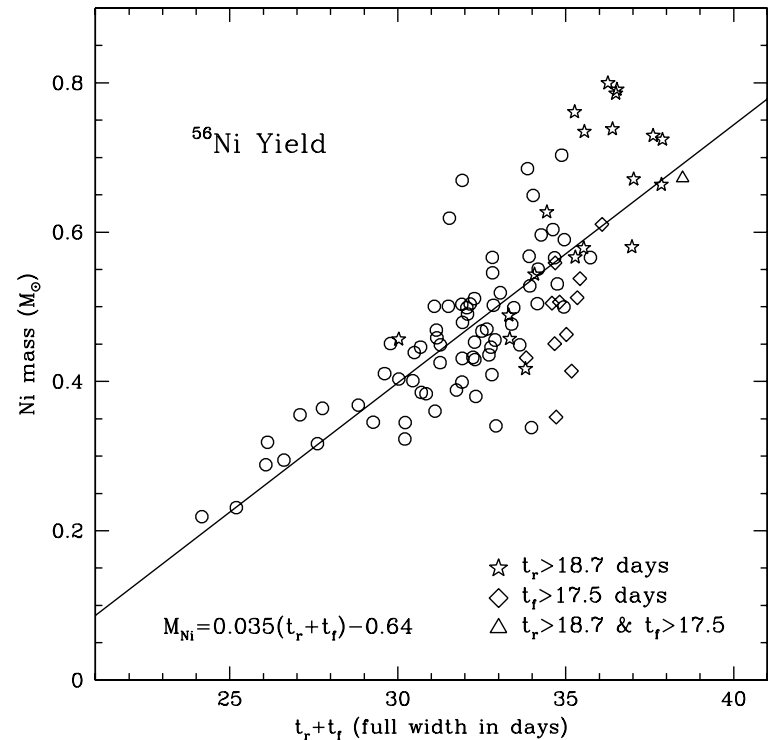

Figure 15. Plot of the derived ${ }^{56} \mathrm{Ni}$ mass for the SDSS-II supernovae vs. the full width (rise plus fall times) of the $B$-band light curve. The ${ }^{56} \mathrm{Ni}$ mass is estimated from Arnett's rule so it is a function of both the peak bolometric luminosity and the rise time. The solid line is a least-squares fit to the points. Supernovae with slow rise times $\left(t_{r}>18.7\right.$ days) are plotted as stars while those with slow declines $\left(t_{f}>17.5\right.$ days) are shown as diamonds. Only one light curve had both a slow rise and decline and it is plotted as a triangle with a full width of 38 days. The supernovae with the largest ${ }^{56} \mathrm{Ni}$ yields $\left(>0.7 M_{\odot}\right)$ have slowly rising light curves while slowly fading events produce more typical amounts ranging between 0.4 and $0.6 M_{\odot}$ of ${ }^{56} \mathrm{Ni}$. Clearly, knowledge of the rise time is critical in understanding the physics of Type Ia events.

implying that the presence of a range in rise times has very little direct impact on cosmological measurements.

\subsection{Estimating ${ }^{56} \mathrm{Ni}$ Yields}

Arnett's rule (Arnett 1982) states that the bolometric luminosity of a SN Ia at maximum light is very close to the instantaneous energy being deposited by the synthesized radioactive elements. This equality has been used by several studies (e.g., Stritzinger \& Leibundgut 2005; Contardo et al. 2000; Howell et al. 2009) to estimate the mass of ${ }^{56} \mathrm{Ni}$ created in the supernova. However, the time between explosion and maximum light is a key parameter in this calculation and it has often been assumed to be a constant 19.5 days. In this paper, we have found that the average rise time is shorter than that estimated in previous studies and it is not strictly linked to the decline speed. This will change the distribution of ${ }^{56} \mathrm{Ni}$ yields when compared with earlier assumptions about rise-time properties. We estimate ${ }^{56} \mathrm{Ni}$ yields using the prescription of Stritzinger \& Leibundgut (2005) but with a simplified technique for measuring the bolometric luminosity. We used the time from explosion to peak flux as our measured risetime values. We have already calculated the extinction-corrected absolute $V$-band magnitudes for the well-observed objects in the SDSS-II sample and we use these to scale an average Type Ia spectrum which we integrate over a wide wavelength range to get quasi-bolometric $U V O I R$ luminosity. For a supernova with absolute magnitude $M_{V}=0.00$ (Vega system) and the Hsiao et al. (2007) spectral template at $B$-band maximum, we find a bolometric flux of $2.04 \times 10^{-5} \mathrm{erg} \mathrm{cm}^{-2} \mathrm{~s}^{-1}$ between 300 and $1000 \mathrm{~nm}$. This approximation is best for normal Type Ia and is not as accurate for sub-luminous events which tend to be more red than normal at maximum. However, Contardo et al. (2000) found that the $V$ band is an excellent indicator of bolometric energy even at late phases when the spectrum has become red. Under these assumptions, the ${ }^{56} \mathrm{Ni}$ yield is simply

$$
M_{\mathrm{Ni}}=\frac{4 \pi 9.52 \times 10^{38} 10^{-M_{V} / 2.5} 2.04 \times 10^{-5}}{\alpha 6.45 \times 10^{43} \exp \left(-t_{r} / 8.8\right)+1.45 \times 10^{43} \exp \left(-t_{r} / 111\right)}\left(M_{\odot}\right),
$$

where $t_{r}$ is the rise time in days and $\alpha$ is a constant describing the accuracy of Arnett's rule (we assume $\alpha=1$ ).

The estimated ${ }^{56} \mathrm{Ni}$ yields for the SDSS-II events range from 0.2 to $0.8 M_{\odot}$, which are typical for this method (Stritzinger et al. 2006). Figure 15 shows the ${ }^{56} \mathrm{Ni}$ yield as a function of the total light curve width $\left(t_{r}+t_{f}\right)$ and there is a strong correlation between these two quantities. Most of the supernovae producing the largest amount of ${ }^{56} \mathrm{Ni}$ are events with $t_{r}>$ 18.7 days (slowest rising 20\%). In contrast, the slowest declining supernovae $\left(t_{f}>17.5\right.$ days $)$ produce average yields of ${ }^{56} \mathrm{Ni}$. This demonstrates the importance of independently measuring the rise time of $\mathrm{SN}$ Ia to understand the physics of the thermonuclear explosion mechanisms.

\section{CONCLUSION}

The SDSS-II supernova sample provides tight constraints on the rise time and shape of SN Ia light curves. The $k$-corrected, median light curves of 391 events shows a $B$-band rise time of 16.8 days and a significantly smaller dispersion on the rise portion of the curve than on the fading side. This implies that the rise time is less impacted by variations in radioactive nickel yield than the fall, as predicted by the Kasen \& Woosley (2007) model light curves. It is clear that the single-stretch parameter commonly used to characterize SN Ia light curves is not capable of capturing the full range of light curve shapes, especially when pre-maximum observations are available.

We selected $105 \mathrm{SNe}$ Ia from the larger sample that had sufficient photometric precision and cadence to yield rise- and fall-time errors of less than 2.0 days. From this set, we find the rise time in the $B$ band is $t_{r}=17.38 \pm 0.17$ days (standard error of the mean). This is significantly different from the rise time of 19.5 days measured from local supernovae (Riess et al. 1999) and high-redshift events (Conley et al. 2006). We find the cause of this discrepancy to be the difference in the fiducial curves used in the analyses and our application of a 2-stretch fitting method that permits the rise and fall to be fit independently. We find rise times ranging from 13 days to 23 days for events with "normal" decline times of around 15 days, demonstrating that the rise and fall are not strictly correlated.

We applied our 2-stretch light curve fitting method to data from SDSS-II to test the conjecture by Strovink (2007) of a bimodal distribution in SN Ia light curve shape. From our 105 high-quality light curves, we are not able to reproduce this bimodal distribution in the difference between rise and fall times. We do find a significant spread in RMF times for the sample, which implies again that the rise and fall are not strictly correlated. This large range in RMF is expected from explosion models that simply vary the radioactive nickel yield.

Contrary to the premise of the single-stretch method, many of the slowest declining $\mathrm{SNe}$ are among the fastest risers. This effect is better modeled through MLCS2k2, as shown by our simulations, and this may be a result of MLCS2k2 being trained on a large number of real nearby SNe Ia. While MLCS2k2 is better than single stretch at representing the observed light curves, the simulations with MLCS2k2 do not capture the full spread in rise times evident for normal-declining $\mathrm{SNe}$ Ia. Specifically, our simulations using MLCS2K2 do not contain normal-declining events that are slow rising. 
We investigated the relation between peak luminosity, light curve shape, and color by minimizing the scatter on the Hubble diagram for the $96 \mathrm{SNe}$ Ia with good rise/fall data and $\Delta m_{15}(B)<1.5$. The Hubble residuals have the smallest scatter when using both rise and fall time as fit parameters. The correlation of rise and fall time with luminosity have the same sign, meaning that the full width of the light curve is the best indicator of luminosity. Twelve models suggested that a second parameter, such as KE, competing with radioactive nickel yield, could be revealed in the rise and fall times and their correlation with luminosity. However, rise and fall times are found to act in concert in the SDSS-II SNe, implying that KE (or other physical parameters that broaden the light curve and lower peak luminosity) is not a major contributor to light curve shape.

Pignata et al. (2008) recently suggested that SNe Ia with high Si II velocity gradients (HVG) may have fast-rising light curves. We are analyzing the spectra of the SDSS sample to see if there is a rise-time, spectral velocity gradient correlation. It is interesting to note that SN $1990 \mathrm{~N}$, a low-redshift supernova with a very slow rise, has a low Si II velocity gradient, but a more complete sample needs to be constructed.

The application of the 2-stretch fitting method we have developed provides a better fit to SN Ia light curves than simply stretching the entire time axis. By independently fitting the rise and fall portions of each light curve, we found that the rise and fall times are not strictly correlated. When correcting SN Ia luminosity for light curve shape, adding the rise-time parameter to the fall time reduces the scatter in the Hubble diagram when compared to using the fall time alone. However, we find that the full width of the light curve (rise plus fall time) minimizes the scatter about the Hubble line as well as using both rise and fall times as independent parameters. This does suggest some danger doing cosmology by combining a low-redshift supernova set dominated by poor pre-maximum sampling with a well-sampled high-redshift set.

Our results show that measurement of rise time as a separate parameter characterizing SN Ia light curve shape may be a useful diagnostic in understanding the progenitors and explosion mechanisms of thermonuclear events. Based on ${ }^{56} \mathrm{Ni}$ yield calculations in Contardo et al. (2000) and Stritzinger \& Leibundgut (2005), our average rise time would result in a $15 \%-20 \%$ reduction in ${ }^{56} \mathrm{Ni}$ yield as compared to models that use a 19.5 day rise time. However, there are also some SNe Ia that have rise times longer than 19.5 days, resulting in a higher ${ }^{56} \mathrm{Ni}$ yield. It is clear that accurate nickel yield estimates require a good measurement of the rise time for individual events. Future supernova searches that provide well-sampled, high-quality data well before maximum light will reveal more about the nature of SN Ia.

Funding for the creation and distribution of the SDSS and SDSS-II has been provided by the Alfred P. Sloan Foundation, the Participating Institutions, the National Science Foundation, the U.S. Department of Energy, the National Aeronautics and Space Administration, the Japanese Monbukagakusho, the Max Planck Society, and the Higher Education Funding Council for England. The SDSS Web site is http://www.sdss.org/.

The SDSS is managed by the Astrophysical Research Consortium for the Participating Institutions. The Participating Institutions are the American Museum of Natural History, Astrophysical Institute Potsdam, University of Basel, Cambridge University, Case Western Reserve University, University of Chicago, Drexel University, Fermilab, the Institute for Advanced Study, the Japan Participation Group, Johns Hopkins University, the Joint Institute for Nuclear Astrophysics, the Kavli Institute for Particle Astrophysics and Cosmology, the Korean Scientist Group, the Chinese Academy of Sciences (LAMOST), Los Alamos National Laboratory, the Max-PlanckInstitute for Astronomy (MPA), the Max-Planck-Institute for Astrophysics (MPiA), New Mexico State University, Ohio State University, University of Pittsburgh, University of Portsmouth, Princeton University, the United States Naval Observatory, and the University of Washington.

This work is based in part on observations made at the following telescopes. The Hobby-Eberly Telescope (HET) is a joint project of the University of Texas at Austin, the Pennsylvania State University, Stanford University, Ludwig-MaximilliansUniversität München, and Georg-August-Universität Göttingen. The HET is named in honor of its principal benefactors, William P. Hobby and Robert E. Eberly. The Marcario Low-resolution Spectrograph is named for Mike Marcario of High Lonesome Optics, who fabricated several optical elements for the instrument but died before its completion; it is a joint project of the Hobby-Eberly Telescope partnership and the Instituto de Astronomía de la Universidad Nacional Autónoma de México. The Apache Point Observatory $3.5 \mathrm{~m}$ telescope is owned and operated by the Astrophysical Research Consortium. We thank the observatory director, Suzanne Hawley, and site manager, Bruce Gillespie, for their support of this project. The Subaru Telescope is operated by the National Astronomical Observatory of Japan. The William Herschel Telescope is operated by the Isaac Newton Group, and the Nordic Optical Telescope is operated jointly by Denmark, Finland, Iceland, Norway, and Sweden, both on the island of La Palma in the Spanish Observatorio del Roque de los Muchachos of the Instituto de Astrofisica de Canarias. Observations at the ESO New Technology Telescope at La Silla Observatory were made under program IDs 77.A-0437, 78.A-0325, and 79.A-0715. Kitt Peak National Observatory, National Optical Astronomy Observatory, is operated by the Association of Universities for Research in Astronomy, Inc. (AURA) under cooperative agreement with the National Science Foundation. The WIYN Observatory is a joint facility of the University of Wisconsin-Madison, Indiana University, Yale University, and the National Optical Astronomy Observatories. The W.M. Keck Observatory is operated as a scientific partnership among the California Institute of Technology, the University of California, and the National Aeronautics and Space Administration. The Observatory was made possible by the generous financial support of the W.M. Keck Foundation. The South African Large Telescope of the South African Astronomical Observatory is operated by a partnership between the National Research Foundation of South Africa, Nicolaus Copernicus Astronomical Center of the Polish Academy of Sciences, the Hobby-Eberly Telescope Board, Rutgers University, Georg-August-Universität Göttingen, University of Wisconsin-Madison, University of Canterbury, University of North Carolina-Chapel Hill, Dartmouth College, Carnegie Mellon University, and the United Kingdom SALT consortium. The Italian Telescopio Nazionale Galileo is operated on the island of La Palma by the Fundacion Galileo Galilei of the Instituto Nazionale di Astrofisica at the Spanish Observatorio del Roque de los Muchachos of the Instituto de Astrofisica de Canarias.

DARK is funded by the Danish National Research Foundation. The Oskar Klein Centre is funded by The Swedish Research Council. Jesper Sollerman is a Royal Academy of 
Sciences Research Fellow supported by a grant from the Knut and Alice Wallenberg Foundation. This research is supported at Rutgers University by the US Department of Energy grant DEFG02-08ER41562 (PI: S.W.J). Work is partially supported by U.S. National Science Foundation through grant AST-0507475 (ESSENCE).

We also thank Dr. Peter Nugent for many helpful and insightful comments.

\section{REFERENCES}

Abazajian, K. N., et al. 2009, ApJS, 182, 543

Aldering, G., Knop, R., \& Nugent, P. 2000, AJ, 119, 2110

Arnett, W. D. 1982, ApJ, 253, 785

Arnett, W. D., Branch, D., \& Wheeler, J. C. 1985, Nature, 314, 337

Arnett, D., \& Livne, E. 1994, ApJ, 427, 330

Astier, P., et al. 2006, A\&A, 447, 31

Barris, B. J., et al. 2006, ApJ, 602, 571

Benetti, S., et al. 2004, MNRAS, 348, 261

Branch, D., Baron, E., Thomas, R. C., Kasen, D., Li, W., \& Filippenko, A. V. 2004, PASP, 116, 903

Conley, A., et al. 2006, AJ, 132, 1707

Contardo, G., Leibundgut, B., \& Vacca, W. D. 2000, A\&A, 359, 876

Eisenstein, D. J., et al. 2007, ApJ, 664, 675

Freedman, W. L., et al. 2001, ApJ, 553, 47

Frieman, J. A., et al. 2008, AJ, 135, 338

Fukugita, M., Ichikawa, T., Gunn, J. E., Doi, M., Shimasaka, K., \& Schneider, D. P. 1996, AJ, 111, 1748

Gallagher, J. S., Garnavich, P. M., Caldwell, N., Kirshner, R. P., Jha, S. W., Li, W., Ganeshalingam, M., \& Filippenko, A. V. 2008, ApJ, 685, 752

Gallagher, J. S., et al. 2005, ApJ, 634, 210

Garg, A., et al. 2007, AJ, 133, 403

Garnavich, P. M., et al. 1998a, ApJ, 493, L53

Garnavich, P. M., et al. 1998b, ApJ, 509, 74

Garnavich, P. M., et al. 2004, ApJ, 613, 1120

Goldhaber, G., et al. 2001, ApJ, 558, 359

Gunn, J. E., et al. 1998, AJ, 116, 3040

Gunn, J. E., et al. 2006, AJ, 131, 2332

Guy, J., et al. 2007, A\&A, 466, 11

Hamuy, M., Phillips, M. M., Suntzeff, N. B., Schommer, R. A., Maza, J., \& Aviles, R. 1996a, AJ, 112, 2391

Hamuy, M., et al. 1996b, AJ, 112, 2398

Hamuy, M., et al. 2003, Nature, 424, 651

Hayden, B., Garnavich, P. M., Jha, S. W., \& Survey, S.-I. S. 2010, BAAS, 41, 362

Hicken, M., Challis, P., Jha, S., Kirshner, R. P., Matheson, T., Modjaz, M., Rest, A., \& Wood-Vasey, W. M. 2009, ApJ, 700, 331

Hicken, M., et al. 2007, ApJ, 669, L17

Hoeflich, P., \& Khokhlov, A. 1996, ApJ, 457, 500

Hoeflich, P., et al. 2010, ApJ, 710, 444

Hogg, D. W., Finkbeiner, D. P., Schlegel, D. J., \& Gunn, J. E. 2001, AJ, 122, 2129

Holtzman, J. A., et al. 2008, AJ, 136, 2306

Howell, D. A., et al. 2006, Nature, 443, 308

Howell, D. A., et al. 2009, ApJ, 691, 661

Hsiao, E. Y., Conley, A., Howell, D. A., Sullivan, M., Pritchet, C. J., Carlberg, R. G., Nugent, P. E., \& Phillips, M. M. 2007, ApJ, 663, 1187

Ivezić, Ż., et al. 2004, Astron. Nachr., 325, 583

Jha, S., Branch, D., Chornock, R., Foley, R. J., Li, W., Swift, B. J., Casebeer, D., \& Filippenko, A. V. 2006, AJ, 132, 189

Jha, S., Riess, A. G., \& Kirshner, R. P. 2007, ApJ, 659, 122

Jha, S., et al. 1999, ApJS, 125, 73
Kasen, D. 2010, ApJ, 708, 1025

Kasen, D., \& Plewa, T. 2007, ApJ, 662, 459

Kasen, D., \& Woosley, S. E. 2007, ApJ, 656, 661

Kessler, R., et al. 2009a, ApJS, 185, 32

Kessler, R., et al. 2009b, PASP, 121, 1028

Khokhlov, A. M. 1991, A\&A, 245, 114

Knop, R. A., et al. 2003, ApJ, 598, 102

Krisciunas, K., et al. 2003, AJ, 125, 166

Lampeitl, H., et al. 2010, MNRAS, 401, 2331

Leibundgut, B. 1989, PhD thesis, Univ. Basel

Lira, P., et al. 1998, AJ, 115, 234

Livio, M. 2000, Type Ia Supernovae: Theory and Cosmology (Cambridge: Cambridge Univ. Press)

Maeda, K., Kawabata, K., Li, W., Tanaka, M., Mazzali, P. A., Hattori, T., Nomoto, K., \& Filippenko, A. V. 2009, ApJ, 690, 1745

Mannucci, F., Della Valle, M., \& Panagia, N. 2006, MNRAS, 370, 773

Miknaitis, G., et al. 2007, ApJ, 666, 674

Pastorello, A., et al. 2007a, MNRAS, 376, 1301

Pastorello, A., et al. 2007b, MNRAS, 377, 1531

Patat, F., et al. 1996, MNRAS, 278, 111

Perlmutter, S., et al. 1997, ApJ, 483, 565

Perlmutter, S., et al. 1998, Nature, 391, 51

Perlmutter, S., et al. 1999, ApJ, 517, 565

Phillips, M. M. 1993, ApJ, 413, L105

Phillips, M. M., et al. 2007, PASP, 119, 360

Pier, J. R., Munn, J. A., Hindsley, R. B., Hennessy, G. S., Kent, S. M., Lupton, R. H., \& Ivezić, Ž. 2003, AJ, 125, 1559

Pignata, G., et al. 2008, MNRAS, 388, 971

Pinto, P. A., \& Eastman, R. G. 2000a, ApJ, 530, 744

Pinto, P. A., \& Eastman, R. G. 2000b, arXiv:astro-ph/0006171

Prieto, J. L., Rest, A., \& Suntzeff, N. B. 2006, ApJ, 647, 501

Prieto, J. L., et al. 2007, AJ, submitted (arXiv:0706.4088)

Pritchet, C. J., Howell, D. A., \& Sullivan, M. 2008, ApJ, 638, L25

Pritchet, C. J., \& The SNLS Collaboration 2005, in ASP Conf. Ser. 339 Observing Dark Energy, ed. S. C. Wolff \& T. R. Lauer (San Francisco, CA: ASP), 60

Riess, A. G., et al. 1998, AJ, 116, 1009

Riess, A. G., et al. 1999, AJ, 118, 2675

Riess, A. G., et al. 2004, ApJ, 607, 665

Riess, A. G., et al. 2005, BAAS, 37, 1460

Riess, A. G., et al. 2007, ApJ, 659, 98

Sako, M., et al. 2008, AJ, 135, 348

Scannapieco, E., \& Bildsten, L. 2005, ApJ, 629, L85

Sekiguchi, M., \& Fukugita, M. 2000, AJ, 120, 1072

Smith, J. A., et al. 2002, AJ, 123, 2121

Sollerman, J., et al. 2009, ApJ, 703, 1374

Stanishev, V., et al. 2007, A\&A, 469, 645

Strovink, M. 2007, ApJ, 671, 1084

Stritzinger, M., \& Leibundgut, B. 2005, A\&A, 431, 423

Stritzinger, M., Mazzali, P. A., Sollerman, J., \& Benetti, S. 2006, A\&A, 460, 793

Stoughton, C., et al. 2002, AJ, 123, 485

Sullivan, M., et al. 2006, ApJ, 648, 868

Timmes, F. X., Brown, E. F., \& Truran, J. W. 2004, Cosmic Explosions in Three Dimensions (Cambridge: Cambridge Univ. Press), 179

Tonry, J. L., et al. 2003, ApJ, 594, 1

Tucker, D. L., et al. 2006, Astron. Nachr., 327, 821

Wood-Vasey, W. M., et al. 2007, ApJ, 666, 694

Woosley, S. E., Kasen, D., Blinnikov, S., \& Sorokina, E. 2007, ApJ, 662, 487

Woosley, S. E., Kerstein, A. R., Sankaran, V., \& Roepke, F. K. 2009, ApJ, 704, 255

York, D. G., et al. 2000, AJ, 120, 1579

Zheng, C., et al. 2008, AJ, 135, 1766 